\title{
Nonlinear systems with fast and slow motions. Changes in the probability distribution for fast motions under the influence of slower ones
}

\author{
Polina S. Landa ${ }^{\mathrm{a}}$ and P.V.E. McClintock ${ }^{\mathrm{b}}$ \\ ${ }^{a}$ Department of Physics, Lomonosov Moscow State University, 119899 Moscow, \\ Russia \\ ${ }^{\mathrm{b}}$ Department of Physics, Lancaster University, Lancaster LA1 4YB, UK
}

\begin{abstract}
The influence of slow processes on the probability distribution of fast random processes is investigated. By reviewing four examples we show that such influence is apparently of a universal character and that, in some cases, this universality is of multifractal form. As our examples we consider theoretically stochastic resonance, turbulent jets with acoustic forcing, and two problems studied experimentally by Shnol' on the influence of the Earth's slow rotation on the probability distribution for the velocities of model Brownian particles and on alpha decay. In the case of stochastic resonance, the slow process is a low frequency, harmonic, external force. In the case of turbulent jets, the slow process is acoustic forcing. In the models based on Shnol's experiments, the slow processes are inertial forces arising from the rotation of the Earth, both about its own axis and about the Sun. It is shown that all of these slow processes cause changes in the probability distributions for the velocities of fast processes interacting with them, and that these changes are similar in form.
\end{abstract}




\section{Contents}

1 Introduction 3

2 Stochastic resonance with white noise 4

3 Control of noise-driven pendulum oscillations by a harmonic force, as a model $\begin{array}{ll}\text { for the control of turbulence by acoustic forcing } & 16\end{array}$

$4 \quad$ Brownian motion in fixed and rotating coordinate systems 28

$\begin{array}{lll}\text { 4.1 Brownian motion in a fixed coordinate system } 28 & 28\end{array}$

4.2 Brownian motion in a rotating coordinate system 30

$5 \quad$ Alpha decay in stationary and rotating coordinate systems 33

5.1 Alpha decay in a stationary coordinate system (classical model) 33

5.2 Influence of the Earth's rotation on alpha decay 43

$\begin{array}{lll}6 & \text { Conclusion } & 45\end{array}$

$\begin{array}{ll}\text { References } & 46\end{array}$ 


\section{Introduction}

The separation of system dynamics into fast and slow motions has been described in numerous papers and books, both for classical [1] and quantum mechanical [2] systems. In most cases, the emphasis has focused on the influence of fast processes on slow ones, as described in detail by Blekhman [1]. A similar separation has often been used for the approximate study of oscillations and waves [3-25,1,26] In this paper we will be interested mainly in the opposite direction of influence (i.e. that of slow processes on fast ones), which has received much less attention, and has mainly been considered in terms of changes in dissipative forces [27] and the influence of a slow harmonic force on fast vibrational processes in the case of vibrational resonance [28-31].

The kinds of phenomena in which we are interested are liable to arise whenever a fast process is modulated by a slow one. Examples may include the unexpected periodicities that occur in alpha and beta decay rates (fast processes), arguably associated with the small changes that occur in the Sun-Earth separation (a slow process) as the Earth moves along its orbit [32] or to radial mode oscillations of the inner solar tachocline separating the radiative zone from the core (another slow process) [33]. Similarly, there are slow changes in the fluctuation spectrum of Brownian motion [34]. It seems that, in all of these cases, this influence manifests as a characteristic change in the probability distribution for the velocity/rate of the fast processes [34,35]. Of particular relevance here, a similar effect was found theoretically while investigating stochastic resonance [36,37], although its importance was not appreciated at the time.

In what follows we illustrate these ideas by consideration of four seemingly quite different physical examples. In Sec. 2 we take the simplest form of stochastic resonance $[36,37]$ and calculate the change in the probability distribution of fast fluctuations under influence of a slow harmonic signal. We show that the way in which it changes is independent of both noise intensity and time-scale, i.e. the process possesses the property of multifractal universality $[38,39]$. Sec. 3 discusses the application of similar ideas to a model of turbulence, where the slow control process modulates the fast turbulent fluctuations. As other examples we consider in Secs. 4 and 5 respectively the influence of the Earth's rotation on 
Brownian motion [40] and on alpha decay rates [41]. In these latter two cases, we assume that the experimental results are valid but hitherto unexplained. Finally, in Sec. 6 we summarise our results and draw conclusions.

\section{Stochastic resonance with white noise}

The notion of stochastic resonance was introduced in 1981 to try to account for the seemingly periodic (with period $T \simeq 100000$ years) onset of ice ages [42,43]. It was appreciated that one possible cause of the phenomenon was the periodic change in the eccentricity of the Earth's orbit, which affects the amount of solar radiation reaching the Earth's surface. Although this variation occurs with just the right period it is, in itself, far too small to cause significant climatic change. However, allowing for the influence of noise and adding it into their initial equation, the authors demonstrated that such changes were in fact possible, because the weak periodic influence could effectively be amplified by the noise, which is what became known as stochastic resonance (SR). Although the question of its applicability to climatology still remains unsettled, the phenomenon of SR became widely known, was intensively researched, and has subsequently been found to manifest in a wide range of other physical and biological contexts.

In the initial investigations of SR, the alternation of ice ages and inter-glacial periods was considered in terms of the Earth's surface temperature, modelled with an equation of motion for a light particle in a bistable potential field disturbed by a weak periodic signal in the presence of additive white noise:

$$
\dot{x}+f(x)=A \cos \omega t+\xi(t)
$$

where $x$ is the particle displacement, $A \cos \omega t$ is a weak periodic force at frequency $\omega$, $f(x)=d U(x) / d x, U(x)$ is a symmetric double-well potential (we will consider the simplest case of such a potential, namely, $\left.U(x)=-x^{2} / 2+x^{4} / 4\right)$, and $\xi(t)$ is white noise of intensity $K$, i.e. $\langle\xi(t) \xi(t+\tau)\rangle=K \delta(\tau)$. Because the frequency of the oscillations in eccentricity is very low, the disturbance $A \cos \omega t$ is assumed to be very slow in comparison with the characteristic time of the noise $\xi(t)$. Thus we have an overdamped, bistable oscillator 
subjected to fast and slow forces.

The power spectrum of a solution of Eq. (2.1) contains [44,45] discrete components that are odd and even harmonics of frequency $\omega$, and continuous constituents caused by the noise $\xi(t)$. We will show (see below) that only the odd harmonics are essential, so that we can write $x(t)$ as

$$
x(t)=s(t)+n(t)
$$

where

$$
\begin{aligned}
& s(t)=\langle x(t)\rangle=\sum_{k=0}^{\infty} B_{2 k+1} \cos \left((2 k+1) \omega t+\psi_{2 k+1}\right), \\
& n(t)=x(t)-s(t), \quad\langle n(t)\rangle=0 .
\end{aligned}
$$

We will refer to the ratio of $B_{1}$ to $A$ as the gain factor, and will denote it by $Q(K, \omega, A)$.

A numerical solution of Eq. (2.1) shows that the gain factor $Q(K, \omega, A)$ and phase shift $\psi(K, \omega, A)$ are nonmonotonic functions of the noise intensity $K: Q$ passes through a maximum at a particular value of $K$ that rises as the signal frequency increases. The dependence of $Q$ on $K$ at fixed signal frequency and amplitude resembles the resonant dependence of an oscillator's amplitude on the external forcing frequency. Because $K$ defines the mean frequency of jumps from one well to the other caused by random transitions through the potential barrier [24], it was initially assumed that the maximum in $Q(K)$ occurs when the signal period $T=2 \pi / \omega$ is equal to double the mean time

$$
T_{\mathrm{tr}}=\frac{\pi}{\sqrt{2}} \exp \left(\frac{2 U_{0}}{K}\right)
$$

of the first transition through potential barrier. It is evident, however, that this assumption is incorrect: otherwise $Q$ would pass through a maximum, not only with changing $K$, but also with changing $\omega$. In reality, $Q$ decreases monotonically as $\omega$ increases.

We now describe the results obtained from a numerical solution of Eq. (2.1). For a sufficiently small amplitude of the harmonic disturbance $A$, and in the absence of noise $\xi(t)$ the oscillations are close to being harmonic at frequency $\omega$ and occur about one of the 
stable equilibrium positions $x_{0}= \pm 1$. When the noise intensity is small, rare jumps occur from the vicinity of one of these equilibrium positions to the vicinity of the other one, and the oscillations become noisy: to discern the presence of the harmonic disturbance without special measures become impossible. As the noise intensity rises, the mean frequency of jumps increases steadily.

One perception of SR is in terms of a noise-induced change in the system parameters, which results in the resonance-like dependence of $Q$ on $K$ in the overdamped oscillator described by Eq. (2.1) [24,36,46]. To calculate the effective (i.e. noise-modified) parameters of the system, we represent a solution of Eq. (2.1) in the form of (2.2), substitute (2.2) into Eq. (2.1) and then split (2.1) into two equations such that one of them describes quantities averaged over the statistical ensemble, and the other describes the deviations from those averaged values. Because at $s=0$ all the odd noise moments $m_{j}=\left\langle n^{j}\right\rangle$ are equal to zero, we can set approximately

$$
m_{3}=a s+b \dot{s},
$$

where $a$ and $b$ are unknown functions of $K$ and $\omega$, which will be found later. Taking this into account we write the equations for $s(t)$ and $n(t)$ as

$$
\begin{aligned}
(1+b) \dot{s}+c s+s^{3} & =A \cos \omega t \\
\dot{n}+\left(3 s^{2}-1\right) n+n^{3}+\left(3 n^{2}-1-c\right) s-b \dot{s} & =\xi(t),
\end{aligned}
$$

where

$$
c=3 m_{2}-1+a
$$

is the effective stiffness, and $b$ is the noise-induced addition to the damping factor.

This division of the initial equation into two equations is similar to the separation of the dynamics into slow and fast motion as suggested in [1]. In contrast, however, here we separate the motions into regular and random parts. As in [1], this splitting is not unique.

It follows from Eq. (2.6) that the variable $s(t)$ can execute oscillations either about zero, if $3 m 2-1+a>0$, or about one of the equilibrium states $s_{0}= \pm\left(1-3 m_{2}-a\right)$, if 
$3 m_{2}-1+a<0$. Oscillations about zero are possible only for $K=0$, i.e. in the absence of noise.

To calculate the moments $m_{j}$ and find $a$ and $b$, we use the Fokker-Planck equation corresponding to the Langevin equation (2.7) which, in the linear approximation with respect to $s(t)$ and $\dot{s}$, is

$$
\frac{\partial w}{\partial t}=\frac{\partial}{\partial n}\left\{\left[n^{3}-n+\left(3 n^{2}-1-c\right) s-b \dot{s}\right] w\right\}+\frac{K}{2} \frac{\partial^{2} w}{\partial n^{2}} \cdot(2.9)
$$

It is convenient to seek a solution of Eq. (2.9) in the form of a sum of three constituents, one of which, $w_{0}$, is dominant (of order 1 ), and the other two, $w_{1}$ and $w_{2}$, are small and of order $\epsilon$, where $\epsilon$ is a conventional small parameter. So, we set

$$
w(n, t)=w_{0}(n)+\epsilon\left(w_{1}(n) s+w_{2}(n) \dot{s}\right)
$$

The first term on the right describes the probability distribution for the fast random process $n(t)$ in the absence of the slow one, and the other two terms define the change in this distribution as a consequence of the slow processes $s(t)$ and $\dot{s}$.

Because in the linear approximation $s(t)$ is a harmonic signal of frequency $\omega$, we have

$$
\ddot{s}(t)=-\omega^{2} s(t)
$$

Taking this into account, substituting (2.10) into Eq. (2.9), restricting ourselves to terms of the first order with respect to $\epsilon$ and equating the coefficients of $s(t)$ and $\dot{s}(t)$, we obtain the following equations for $w_{0}(n), w_{1}(n)$ and $w_{2}(n)$ :

$$
\begin{aligned}
& \frac{K}{2} \frac{\partial w_{0}}{\partial n}+\left(n^{3}-n\right) w_{0}=0 \\
& w_{1}-\left(\frac{d}{d n}\left(\left(n^{3}-n\right) w_{2}\right)+\frac{K}{2} \frac{d^{2} w_{2}}{d n^{2}}\right)=b \frac{d w_{0}}{d n} \\
& -\omega^{2} w_{2}-\left(\frac{d}{d n}\left(\left(n^{3}-n\right) w_{1}\right)+\frac{K}{2} \frac{d^{2} w_{1}}{d n^{2}}\right)=-\frac{d}{d n}\left(\left(3 n^{2}-1-c\right) w_{0}\right) .
\end{aligned}
$$




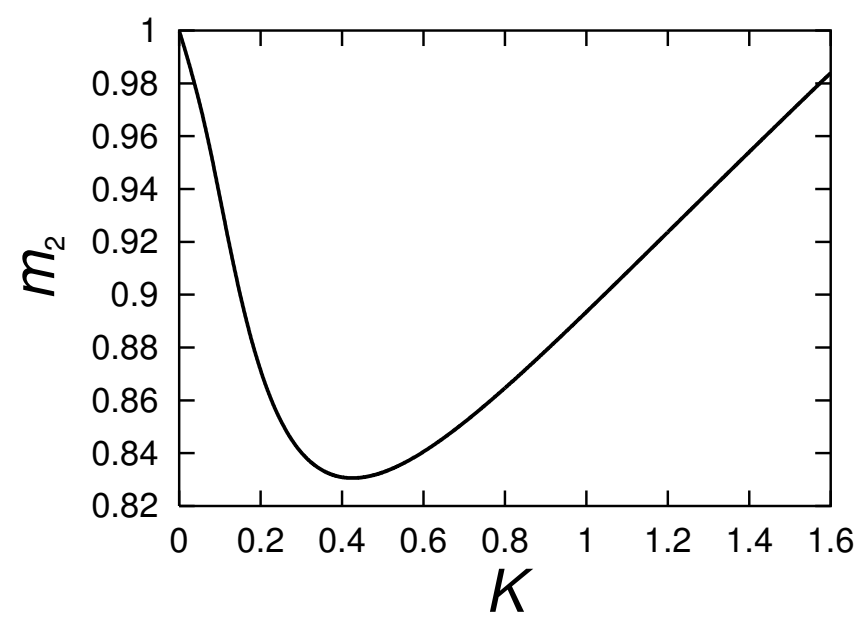

Fig. 1. The dependence on noise intensity $K$ of the noise variance $m_{2}$.

A solution of Eq. (2.12) vanishing for $n= \pm \infty$ is

$$
w_{0}(n)=C(K) \exp \left(\frac{n^{2}}{K}-\frac{n^{4}}{2 K}\right),
$$

where the normalization constant $C(K)=0.008078503537$ for $K=0.1$ and $C(K)=$ 0.06591872565 for $K=0.2$. We see that $w_{0}(n)$ is a narrow, even, function whose width decreases as $K$ increases.

Knowing $w_{0}(n)$ we can find the variance of $n$ as a function of $K$ :

$$
m_{2}(K)=\int_{-\infty}^{\infty} n^{2} w_{0}(n) d n .
$$

which is plotted in Fig. 1. We see that there is a value of $K$ for which the noise variance $m_{2}=\left\langle n^{2}\right\rangle$ is minimal.

Integrating Eqs. (2.13) over $n$ from $-\infty$ to $\infty$ and taking into account that functions $w_{0}(n), w_{1}(n)$ and $w_{2}(n)$ must vanish for $|n| \rightarrow \infty$, we find

$$
\int_{-\infty}^{\infty} w_{1,2}(n) d n=0 .
$$

It is easily seen that this condition means that the functions $w_{1}(n)$ and $w_{2}(n)$ are odd, yielding the initial conditions

$$
w_{1,2}(0)=0 .
$$


From the condition $\langle n\rangle=0$ and the expression (2.5) for the third moment, and in view of (2.8), we find the following relationships :

$$
\begin{aligned}
& \int_{-\infty}^{\infty} n w_{1}(n) d n=0, \quad \int_{-\infty}^{\infty} n w_{2}(n) d n=0, \\
& \int_{-\infty}^{\infty} n^{3} w_{1}(n) d n=c+1-3 m_{2}, \quad \int_{-\infty}^{\infty} n^{3} w_{2}(n) d n=b,
\end{aligned}
$$

where $w_{1}(n)$ and $w_{2}(n)$ are solutions of Eqs. (2.13). For these relationships (2.18) to be true simultaneously, the functions $w_{1}(n)$ and $w_{2}(n)$ must contain arbitrary constants.

The general solution of the inhomogeneous equations (2.13) is

$$
w_{1}(n)=w_{1 \mathrm{in}}(n)+w_{1 \mathrm{~h}}(n), \quad w_{2}(n)=w_{2 \mathrm{in}}(n)+w_{2 \mathrm{~h}}(n),
$$

where $w_{1 \text { in }}(n)$ and $w_{2 \text { in }}(n)$ are partial solutions of the inhomogeneous equations (2.13), which can conveniently be represented as a sum of three components:

$$
\begin{aligned}
& w_{1 \text { in }}(n)=w_{11 \text { in }}(n)+w_{12 \text { in }}(n) c+w_{13 \text { in }}(n) b, \\
& w_{2 \text { in }}(n)=w_{21 \text { in }}(n)+w_{22 \text { in }}(n) c+w_{2 \operatorname{in}}(n) b ;
\end{aligned}
$$

and $w_{1 \mathrm{~h}}(n)$ and $w_{2 \mathrm{~h}}(n)$ represent partial solutions of the homogeneous Eqs. (2.13), which can conveniently be represented as a linear combination of two independent partial solutions:

$$
w_{1 \mathrm{~h}}(h)=C_{1} w_{11 \mathrm{~h}}(n)+C_{2} w_{12 \mathrm{~h}}(n), \quad w_{2 \mathrm{~h}}(n)=C_{1} w_{21 \mathrm{~h}}(n)+C_{2} w_{22 \mathrm{~h}}(n) .
$$

Integrating Eqs. (2.13) over $n$ from 0 to $\infty$, and in view of (2.19), (2.20) and (2.21), we obtain the following relationships:

$$
\begin{aligned}
& \int_{0}^{\infty} w_{11 \text { in }}(n) d n-\left.\frac{K}{2} \frac{d w_{21 \text { in }}(n)}{d n}\right|_{n=0}=0 \\
& \int_{0}^{\infty} w_{12 \text { in }}(n) d n-\left.\frac{K}{2} \frac{d w_{22 \text { in }}(n)}{d n}\right|_{n=0}=0
\end{aligned}
$$




$$
\begin{aligned}
& \int_{0}^{\infty} w_{13 \operatorname{in}}(n) d n-\left.\frac{K}{2} \frac{d w_{23 \operatorname{in}}(n)}{d n}\right|_{n=0}=-w_{0}(0), \\
& -\omega^{2} \int_{0}^{\infty} w_{21 \text { in }}(n) d n-\left.\frac{K}{2} \frac{d w_{11 \text { in }}(n)}{d n}\right|_{n=0}=-w_{0}(0), \\
& -\omega^{2} \int_{0}^{\infty} w_{22 \text { in }}(n) d n-\left.\frac{K}{2} \frac{d w_{12 \text { in }}(n)}{d n}\right|_{n=0}=-w_{0}(0), \\
& -\omega^{2} \int_{0}^{\infty} w_{23 \text { in }}(n) d n-\left.\frac{K}{2} \frac{d w_{13 \text { in }}(n)}{d n}\right|_{n=0}=0,
\end{aligned}
$$

where $w_{0}(0)=C(K)$.

These relationships allow us in principle to find the missing initial conditions for Eqs.(2.13). From (2.22) we find:

$$
\begin{aligned}
& \left.\frac{d w_{11 \text { in }}(n)}{d n}\right|_{n=0}=\frac{2 C(K)}{K}-\frac{2 \omega^{2}}{K} \int_{0}^{\infty} w_{21 \text { in }}(n) d n=0, \\
& \left.\frac{d w_{12 \text { in }}(n)}{d n}\right|_{n=0}=\frac{2 C(K)}{K}-\frac{2 \omega^{2}}{K} \int_{0}^{\infty} w_{22 \text { in }}(n) d n \\
& \left.\frac{d w_{13 \text { in }}(n)}{d n}\right|_{n=0}=-\frac{2 \omega^{2}}{K} \int_{0}^{\infty} w_{23 \text { in }}(n) d n \\
& \left.\frac{d w_{21 \text { in }}(n)}{d n}\right|_{n=0}=\frac{2}{K} \int_{0}^{\infty} w_{11 \text { in }}(n) d n \\
& \left.\frac{d w_{22 \text { in }}(n)}{d n}\right|_{n=0} ^{\infty}=\frac{2}{K} \int_{0}^{\infty} w_{12 \text { in }}(n) d n \\
& \left.\frac{d w_{23 \text { in }}(n)}{d n}\right|_{n=0}=\frac{2 C(K)}{K}+\frac{2}{K} \int_{0}^{\infty} w_{13 \text { in }}(n) d n .
\end{aligned}
$$

It should be noted that these initial conditions are implicit.

Substituting (2.18) into equations (2.13), taking account of (2.20) and (2.21), and equating the coefficients of $c, b, C_{1}$ and $C_{2}$, we find the following differential equations for $w_{11 \text { in }}(n)$, $w_{12 \text { in }}(n), w_{13 \text { in }}(n), w_{21 \text { in }}(n), w_{22 \text { in }}(n), w_{23 \text { in }}(n), w_{11 \mathrm{~h}}(n), w_{12 \mathrm{~h}}(n), w_{21 \mathrm{~h}}(n)$ and $w_{22 \mathrm{~h}}(n)$ : 


$$
\begin{aligned}
& w_{11 \text { in }}(n)-\frac{d}{d n}\left(\left(n^{3}-n\right) w_{21 \text { in }}(n)\right)-\frac{K}{2} \frac{d^{2} w_{21 \text { in }}(n)}{d n^{2}}=0 \\
& w_{12 \text { in }}(n)-\frac{d}{d n}\left(\left(n^{3}-n\right) w_{22 \text { in }}(n)\right)-\frac{K}{2} \frac{d^{2} w_{22 \text { in }}(n)}{d n^{2}}=0 \\
& w_{13 \text { in }}(n)-\frac{d}{d n}\left(\left(n^{3}-n\right) w_{23 \text { in }}(n)\right)-\frac{K}{2} \frac{d^{2} w_{22 \text { in }}(n)}{d n^{2}}=\frac{d w_{0}(n)}{d n} \\
& -\omega^{2} w_{21 \text { in }}(n)-\frac{d}{d n}\left(\left(n^{3}-n\right)\left(w_{11 \text { in }}(n)\right)-\frac{K}{2} \frac{d^{2} w_{11 \text { in }}(n)}{d n^{2}}=-\frac{d}{d n}\left(\left(3 n^{2}-1\right) w_{0}(n)\right)\right. \\
& -\omega^{2} w_{22 \text { in }}(n)-\frac{d}{d n}\left(\left(n^{3}-n\right)\left(w_{12 \text { in }}(n)\right)-\frac{K}{2} \frac{d^{2} w_{12 \text { in }}(n)}{d n^{2}}=\frac{d w_{0}(n)}{d n}\right. \\
& -\omega^{2} w_{23 \text { in }}(n)-\frac{d}{d n}\left(\left(n^{3}-n\right)\left(w_{13 \text { in }}(n)\right)-\frac{K}{2} \frac{d^{2} w_{13 i n}(n)}{d n^{2}}=0\right. \\
& w_{11 \mathrm{~h}}(n)-\frac{d}{d n}\left(\left(n^{3}-n\right) w_{21 \mathrm{~h}}(n)\right)-\frac{K}{2} \frac{d^{2} w_{21 \mathrm{~h}}(n)}{d n^{2}}=0 \\
& w_{12 \mathrm{~h}}(n)-\frac{d}{d n}\left(\left(n^{3}-n\right) w_{22 \mathrm{~h}}(n)\right)-\frac{K}{2} \frac{d^{2} w_{22 \mathrm{~h}}(n)}{d n^{2}}=0 \\
& -\omega^{2} w_{21 \mathrm{~h}}(n)-\frac{d}{d n}\left(\left(n^{3}-n\right)\left(w_{11 \mathrm{~h}}(n)\right)-\frac{K}{2} \frac{d^{2} w_{11 \mathrm{~h}}(n)}{d n^{2}}=0\right. \\
& -\omega^{2} w_{22 \mathrm{~h}}(n)-\frac{d}{d n}\left(\left(n^{3}-n\right)\left(w_{12 \mathrm{~h}}(n)\right)-\frac{K}{2} \frac{d^{2} w_{12 \mathrm{~h}}(n)}{d n^{2}}=0\right.
\end{aligned}
$$

Equations (2.25) must be solved with the initial conditions (2.17) and (2.24), whereas (2.26) may be solved with any initial conditions.

To solve Eqs. (2.25) with the implicit initial conditions (2.24), we introduce new variables

$$
\begin{aligned}
& \left.\left.W 11(n), n)=\int_{0}^{n} w_{11 \text { in }}(n) d n, \quad W 12(n), n\right)=\int_{0}^{n} w_{12 \text { in }}(n) d n, \quad W 13(n), n\right)=\int_{0}^{n} w_{13 \text { in }}(n) d n, \\
& \left.\left.\left.W_{21}(n), n\right)=\int_{0}^{n} w_{21 \text { in }}(n) d n, \quad W_{22}(n), n\right)=\int_{0}^{n} w_{22 \text { in }}(n) d n, \quad W_{23}(n), n\right)=\int_{0}^{n} w_{23 \text { in }}(n) d n .
\end{aligned}
$$

Subject to these new variables, equations (2.25) with initial conditions (2.24) become

$$
\begin{aligned}
& w_{11 \text { in }}(n)-\frac{d}{d n}\left(\left(n^{3}-n\right) w_{21 \text { in }}(n)\right)-\frac{K}{2} \frac{d^{2} w_{21 \text { in }}(n)}{d n^{2}}=0, \\
& w_{12 \text { in }}(n)-\frac{d}{d n}\left(\left(n^{3}-n\right) w_{22 \text { in }}(n)\right)-\frac{K}{2} \frac{d^{2} w_{22 \text { in }}(n)}{d n^{2}}=0
\end{aligned}
$$




$$
\begin{aligned}
& w_{13 \text { in }}(n)-\frac{d}{d n}\left(\left(n^{3}-n\right) w_{23 \text { in }}(n)\right)-\frac{K}{2} \frac{d^{2} w_{23 \text { in }}(n)}{d n^{2}}=\frac{d w_{0}(n)}{d n} \\
& -\omega^{2} w_{21 \text { in }}(n)-\frac{d}{d n}\left(\left(n^{3}-n\right)\left(w_{11 \text { in }}(n)\right)-\frac{K}{2} \frac{d^{2} w_{11 \text { in }}(n)}{d n^{2}}=-\frac{d}{d n}\left(\left(3 n^{2}-1\right) w_{0}(n)\right)\right. \\
& -\omega^{2} w_{22 \text { in }}(n)-\frac{d}{d n}\left(\left(n^{3}-n\right)\left(w_{12 \text { in }}(n)\right)-\frac{K}{2} \frac{d^{2} w_{12 \text { in }}(n)}{d n^{2}}=\frac{d w_{0}(n)}{d n}\right. \\
& -\omega^{2} w_{23 \text { in }}(n)-\frac{d}{d n}\left(\left(n^{3}-n\right)\left(w_{13 \text { in }}(n)\right)-\frac{K}{2} \frac{d^{2} w_{13 \text { in }}(n)}{d n^{2}}=0\right. \\
& \frac{d W_{11}(n)}{d n}=w_{11 \text { in }}(n), \quad \frac{d W_{12}(n)}{d n}=w_{12 \text { in }}(n), \quad \frac{d W_{13}(n)}{d n}=w_{13 \text { in }}(n) \\
& \frac{d W_{21}(n)}{d n}=w_{21 \text { in }}(n), \quad \frac{d W_{22}(n)}{d n}=w_{22 \text { in }}(n), \quad \frac{d W_{23}(n)}{d n}=w_{23 \text { in }}(n)
\end{aligned}
$$

$$
\begin{aligned}
& w_{11 \text { in }}(0)=w_{12 \text { in }}(0)=w_{13 \text { in }}(0)=w_{21 \text { in }}(0)= \\
& \left.\frac{d w_{11 \text { in }}(n)}{d n}\right|_{n=0}=\frac{2 C(K)}{K}-\frac{2 \omega^{2}}{K} W_{21}(\infty), \\
& \left.\frac{d w_{12 \text { in }}(n)}{d n}\right|_{n=0}=\frac{2 C(K)}{K}-\frac{2 \omega^{2}}{K} W_{22}(\infty), \\
& \left.\frac{d w_{13 \text { in }}(n)}{d n}\right|_{n=0}=\frac{2 C(K)}{K}-\frac{2 \omega^{2}}{K} W_{23}(\infty), \\
& \left.\frac{d w_{21 \text { in }}(n)}{d n}\right|_{n=0}=\frac{2}{K} W_{11}(\infty), \\
& \left.\frac{d w_{22 \text { in }}(n)}{d n}\right|_{n=0}=\frac{2}{K} W_{12}(\infty), \\
& \left.\frac{d w_{23 \text { in }}(n)}{d n}\right|_{n=0}=-\frac{2}{K} W_{13}(\infty) .
\end{aligned}
$$

The functions $w_{11 \text { in }}(n), w_{21 \text { in }}(n), w_{12 \text { in }}(n), w_{22 \text { in }}(n), w_{13 \text { in }}(n), w_{23 \text { in }}(n)$ for $\omega=0.1$ are plotted in Fig. 2 for (1) $K=0.1$ and (2) $K=0.2$.

Fig. 3 illustrates the form of the partial solutions of homogeneous Eqs. (2.26).

Using (2.19) again by substituting it into (2.18), and taking account of (2.20), we obtain the following equations for the unknowns $c, b, C_{1}$ and $C_{2}$ : 

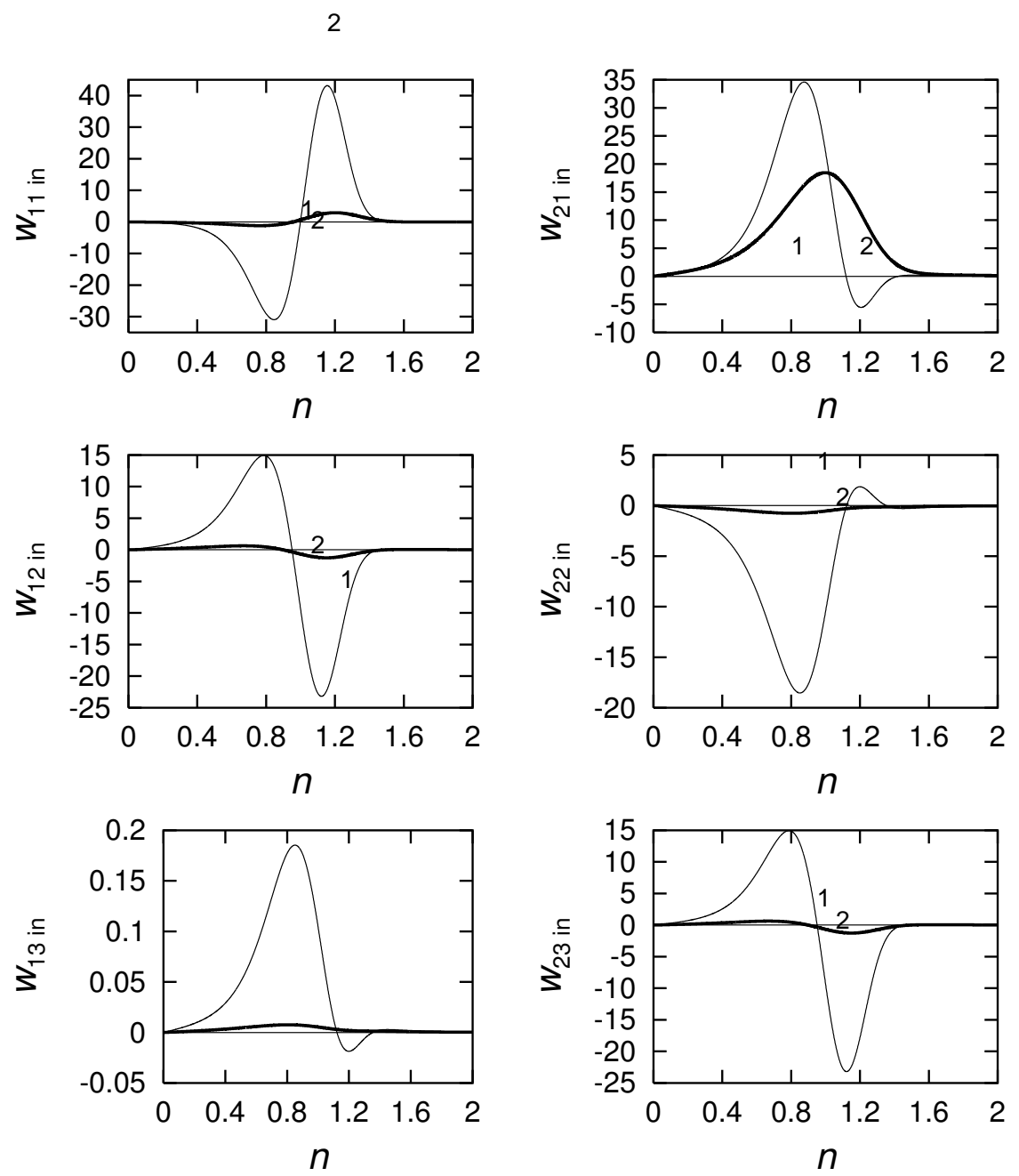

Fig. 2. Solutions of Eqs. (2.28) with initial conditions (2.29) for (1) $\omega=0.1, K=0.1$, and (2) $\omega=0.1, K=0.2$.

$\int_{-\infty}^{\infty}\left(w_{11 \text { in }}+w_{12 \text { in }} c+w_{13 \mathrm{in}} b+w_{11 \mathrm{~h}} C_{1}+w_{12 \mathrm{~h}} C_{2}\right) n d n=0$,

$\int_{-\infty}^{\infty}\left(w_{21 \mathrm{in}}+w_{22 \mathrm{in}} c+w_{23 \mathrm{in}} b+w_{21 \mathrm{~h}} C_{1}+w_{22 \mathrm{~h}} C_{2}\right) n d n=0$,

$\int_{-\infty}^{\infty}\left(w_{11 \text { in }}+w_{12 \mathrm{in}} c+w_{13 \mathrm{in}} b+w_{11 \mathrm{~h}} C_{1}+w_{12 \mathrm{~h}} C_{2}\right) n^{3} d n=c+1-3 m_{2}$

$\int_{-\infty}^{\infty}\left(w_{21 \mathrm{in}}+w_{22 \mathrm{in}} c+w_{23 \mathrm{in}} b+w_{21 \mathrm{~h}} C_{1}+w_{22 \mathrm{~h}} C_{2}\right) n^{3} d n=b$.

Solving Eqs. (2.30), we find $c, b, C_{1}, C_{2}$ and $a$ (the value of $a$ is connected with $c$ and $b$ by relation (2.8)). 

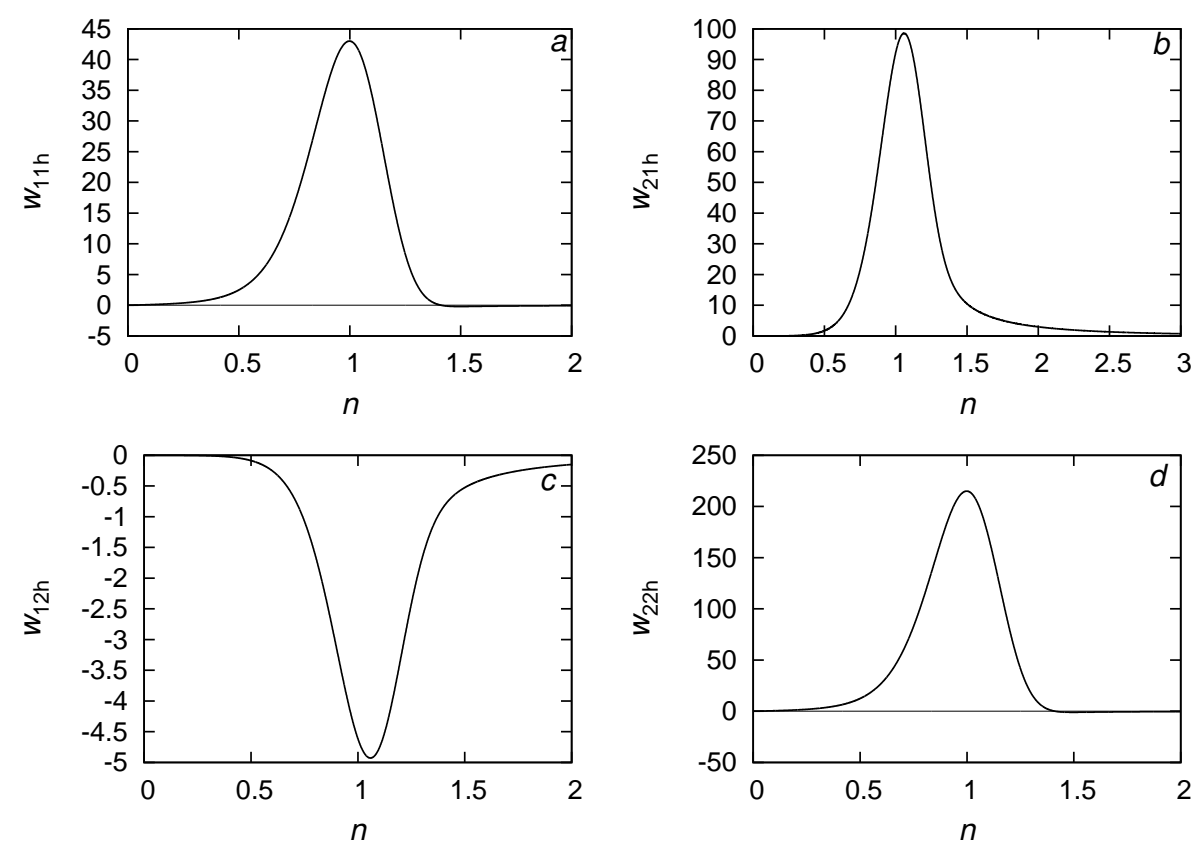

Fig. 3. Partial solutions $w_{11 \mathrm{~h}}(n), w_{21 \mathrm{~h}}(n), w_{12 \mathrm{~h}}(n)$, and $w_{22 \mathrm{~h}}(n)$ with initial conditions $w_{11 \mathrm{~h}}(0)=w_{21 \mathrm{~h}}(0)=w_{12 \mathrm{~h}}(0)=w_{22 \mathrm{~h}}(0)=d w_{12 \mathrm{~h}}(n) /\left.d n\right|_{n=0}=d w_{22 \mathrm{~h}}(n) /\left.d n\right|_{n=0}=0$, $d w_{11 \mathrm{~h}}(n) /\left.d n\right|_{n=0}=1, d w_{22 \mathrm{~h}}(n) /\left.d n\right|_{n=0}=5$ for $\omega=0.1$ and $K=0.1$

As examples, we give the values of $c, b, C_{1}, C_{2}$ and $a$, for $\omega=0.1, K=0.1$, and for $\omega=0.1, K=0.2$ :

- $K=0.1, c=2.332740339, b=8.249221939, a=0.522238519, C_{1}=0.07980201962$, $C_{2}=-0.03353573593$

- $K=0.2, c=-2.718077702, b=13.22379602, a=-4.332166425, C_{1}=-1.080447497$, $C_{2}=0.2717058811$.

It follows from our results that the effective values of $c, b$ and $a$ depend essentially on the signal frequency $\omega$ and of the noise intensity $K$.

Knowing $c, b, w_{11 \text { in }}(n), w_{12 \text { in }}(n), w_{13 \text { in }}(n), w_{21 \text { in }}(n), w_{22 \text { in }}(n), w_{23 \text { in }}(n), w_{11 \mathrm{~h}}(n), w_{12 \mathrm{~h}}(n)$, $w_{21 \mathrm{~h}}(n)$ and $w_{22 \mathrm{~h}}(n)$ we use the formulæ $(2.10)$ and (2.19) to calculate the complete probability distribution $w(n, t)$ and its change $\Delta w(n, t)$ due to the slow forcing, which are defined by

$$
\begin{aligned}
& w(n, t)=w_{0}(n)+\Delta w(n, t), \\
& \Delta w(n, t)=\epsilon\left(w_{1}(n) s(t)+w_{2}(n) \dot{s}\right),
\end{aligned}
$$



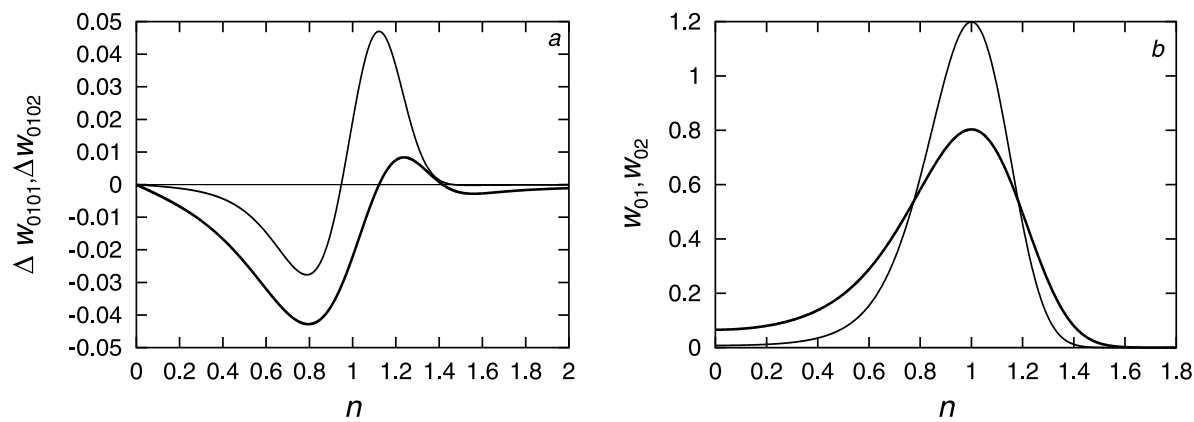

Fig. 4. a) The dependences of $\Delta w(n, t)$ on $n$ for $\omega=0.1, A=0.1, K=0.1$ (thin line) and $\omega=0.1, A=0.1, K=0.2$ (bold line); (b) the dependences of $w(n, t)$ for the same values of $K$.

where $w_{1}(n)$ and $w_{2}(n)$ are defined by (2.20). We will do this on the assumption that the initial conditions for Eqs. (2.26) are equal to zero. In this case $w_{11 \mathrm{~h}}(n), w_{12 \mathrm{~h}}(n), w_{21 \mathrm{~h}}(n)$ and $w_{22 h}(n)$ are also equal to zero and equations (2.31) and (2.32) can be rewritten as

$$
\begin{aligned}
& w(n, t)=w_{0}(n)+\Delta w(n, t), \\
& \Delta w(n, t)=\epsilon\left(w_{11 \text { in }}(n)+c w_{12 \text { in }}(n)+b w_{13 \text { in }}(n)\right) A \cos \omega t \\
& -\omega^{2}\left(w_{21 \text { in }}(n)+c w_{22 \text { in }}(n)+b w_{23 \text { in }}(n)\right) A \sin \omega t .
\end{aligned}
$$

We see that both the complete probability distribution $w(n, t)$ and its change $\Delta w(n, t)$ depend periodically on time.

Examples of the dependences of $w(n, t)$ and $\Delta w(n, t)$ on $n$ for $\omega=0.1, A=0.1, K=0.1$ (1) and $\omega=0.1, A=0.1, K=0.2$ are shown in Figs. 4. We see that the slow signal $s(t)$ changes essentially the form of the probability distribution and decrease its swing.

Thus this simple example of stochastic resonance shows that a slow regular (periodic) forcing of the noisy system changes the probability distribution for the fast motion component i.e. the noise. The size of the change depends on the amplitude and frequency of the slow forcing. 


\section{Control of noise-driven pendulum oscillations by a harmonic force, as a model for the control of turbulence by acoustic forcing}

During the 1960s and 70s researchers in the Russian Central Aerodynamical Institute (TSAGI) proposed and demonstrated a method of controlling turbulence in submerged jets by the application of an acoustic wave $[47,48]$. This process was subsequently investigated in more detail by many other researchers [12-18,49,20,22,23,50,51]; the underlying mechanism can be considered as a manifestation of stochastic resonance [52].

The main results of the enterprise were as follows. In the case of a low-frequency harmonic acoustic action at a frequency $f$, corresponding to Strouhal numbers in the range from $0.2-0.6$, the longitudinal and radial velocity pulsations rise steeply, independently of the direction of the jet, provided that the amplitudes of the longitudinal and radial components of the oscillatory velocity in the sound wave on the jet axis near the nozzle amount to $0.05-2 \%$ of $U_{0}$, where $U_{0}$ is the jet velocity near the nozzle. For the effects to occur, the acoustic wave amplitude must exceed a certain threshold value. As the wave amplitude increases beyond this threshold, there is an intensification of turbulent intermixing followed by saturation: further increase of the acoustic wave amplitude has little or no effect on turbulent processes in the jet.

For high-frequency acoustic action on a jet at a frequency corresponding to Strouhal numbers in the range $1.5-5$, the vortices in the jet mixing layer become smaller, resulting in the attenuation of turbulent intermixing, a reduction in the thickness of the mixing layer, a lengthening of the initial part, and a decrease in entrainment. Longitudinal and radial velocity pulsations at the jet axis are substantially reduced in the process. In contrast to the case of low-frequency action, high-frequency action on the jet does not result in saturation as the action amplitude increases; moreover, an increase in amplitude beyond a certain value causes, not suppression of turbulence, but its intensification.

The latter feature is illustrated by the experimental results shown in Fig. 5 [53,26], which demonstrate that the suppression of turbulence by an acoustic wave of fixed amplitude depends nonmonotonically on its frequency: it is maximal at a certain value of the action 


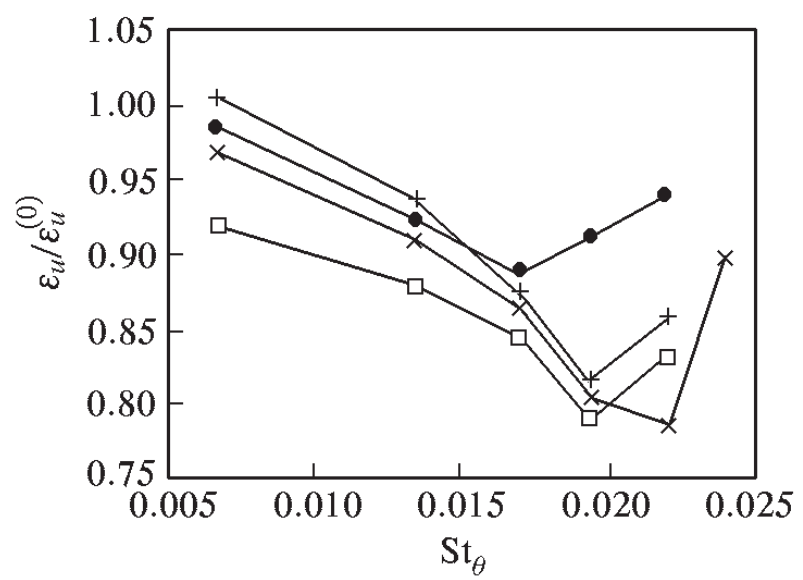

Fig. 5. The experimental dependences on the Strouhal number $\mathrm{St}_{\vartheta}=(\vartheta / D) \mathrm{St}$ of the suppression factor $\epsilon_{u} / \epsilon_{u}^{(0)}$, where $\epsilon_{u}^{(0)}$ is the relative intensity of the longitudinal velocity pulsations in the absence of acoustic forcing, for $x / \vartheta=200$. The plots are constructed for four values of the amplitude of the oscillatory velocity in the acoustic wave, namely $0.5 \%$ of $U_{0}$ (circles), $2.5 \%$ (pluses), $3.5 \%$ (crosses) and 4.5\% (squares). After [36].

frequency depending on the wave amplitude.

It should be noted that these effects are observed quite generally for jets, over a wide range of Reynolds numbers $\left(10^{2}<\operatorname{Re}<10^{6}\right)$. As the intensity of the low-frequency acoustic action on the jet increases, its initial part decreases in length to the point of disappearance [12]. The evolution of the shape of the jet pulsations, and of their power spectra with increasing relative distance from the nozzle exit, as found experimentally, are shown in Fig. 6.

Similar effects are also observed when the periodic action on the jet is effected by other means, e.g. by longitudinal or radial vibration of the nozzle, or by providing a pulsating rate of fluid outflow from the nozzle [18]. To calculate the effects indicated mentioned above, we can make use of an unexpected analogy between turbulent processes in submerged jets and the noise-induced oscillations of a pendulum with a randomly-vibrated suspension axis [54-56,23,26,25,36]. We believe that this analogy arises because the onset of turbulence in jets is a noise-induced phase transition, and the pendulum with a randomly vibrated suspension axis is a paradigmatic model of such a transition [36]. 
When additive noise is neglected, the equation describing the pendulum oscillations is:

$$
\ddot{\varphi}+2 \beta\left(1+\alpha \dot{\varphi}^{2}\right) \dot{\varphi}+\omega_{0}^{2}(1+\xi(t)) \sin \varphi=0,
$$

where $\varphi$ is the pendulum's angular deviation from its equilibrium position, $2 \beta\left(1+\alpha \dot{\varphi}^{2}\right) \dot{\varphi}$ is proportional to the moment of the friction force which is assumed to be nonlinear, $\omega_{0}$ is the natural frequency of small oscillations, and $\xi(t)$ is a comparatively wide-band random process with nonzero power spectral density at the frequency $2 \omega_{0}$.

When the intensity of the suspension axis vibration ${ }^{1}$ exceeds a certain critical value proportional to the friction factor $\beta$, excitation of pendulum oscillations occurs, and the variance of the pendulum angular deviation becomes nonzero.

It can be seen from Fig. 7 that, close to the excitation threshold, the pendulum oscillations possess the property of on-off intermittency. This notion was introduced by Platt et al. [57], although a similar phenomenon had been considered earlier by Fujisaka et al. [58]. It was noted in [57] that intermittency of this kind is similar to the intermittency in turbulent flows. It is important that on-off intermittency is possible, not only in dynamical systems, but in stochastic ones as well [59]. External manifestations of on-off intermittency are similar to ordinary intermittency [60], i.e. over prolonged periods the pendulum oscillates in the immediate vicinity of its equilibrium position ("laminar phases"); these slight oscillations alternate with short random bursts of larger amplitude ("turbulent phases"). Further above the excitation threshold, the durations of the laminar phases decrease and those of the turbulent ones increase, with the laminar phases ultimately disappearing altogether [56]. The variance of the pendulum's angular deviation increases in the process. Comparing the evolution of the power spectra shown in Figs. 6 and 7, we can see that they have much in common: the response of the pendulum to vibration of its suspension axis is evidently very similar to the response of a jet to an acoustic force. For example, in the case when the intensity of the random suspension axis vibration is close to its threshold value, the dependence of the intensity of pendulum oscillations on the frequency of the

\footnotetext{
$\overline{1}$ By intensity of the suspension axis vibration we mean the spectral density of $\xi(t)$ at frequency $2 \omega_{0}\left(\kappa\left(2 \omega_{0}\right)\right)$.
} 

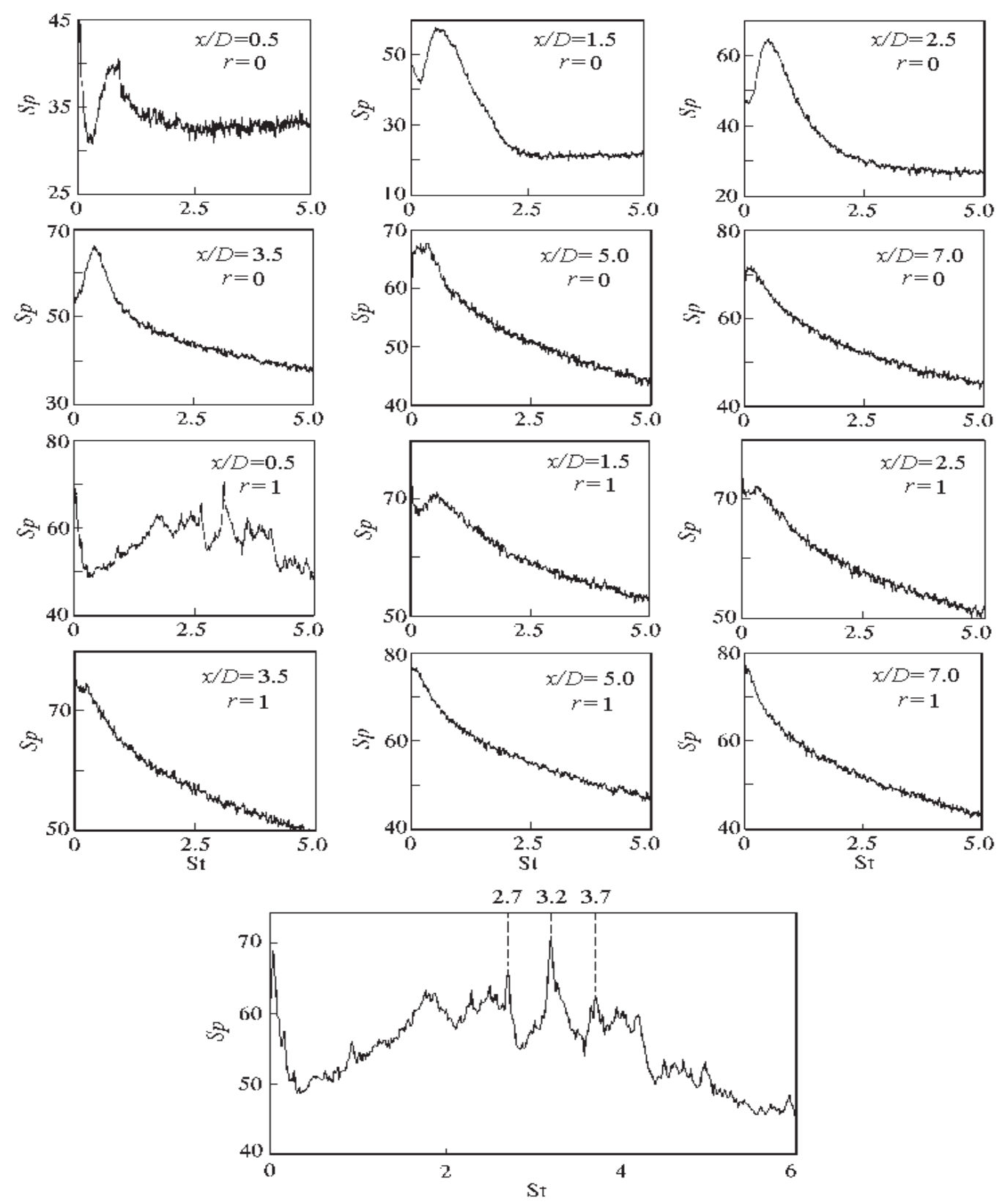

Fig. 6. Experimental results for turbulence from a submerged jet. Examples of the evolution of spectral density $S p$ (in decibels) of the velocity pulsations $u$ with relative distance from the nozzle exit $x / D$ along the jet axis $(r=0)$ and along a line offset by $R$ from the axis $(r=1)$. At the bottom the spectral density for $x / D=0.5, r=1$ is shown over a wide scale. After [36].

additional harmonic forcing is of a resonant character, very much like a jet subject to an acoustic force (cf. Figs. 6 and 7).

The response of the pendulum to the small additional harmonic force (vibration of the suspension axis) is closely similar to the response of the jet to an acoustic force. For 

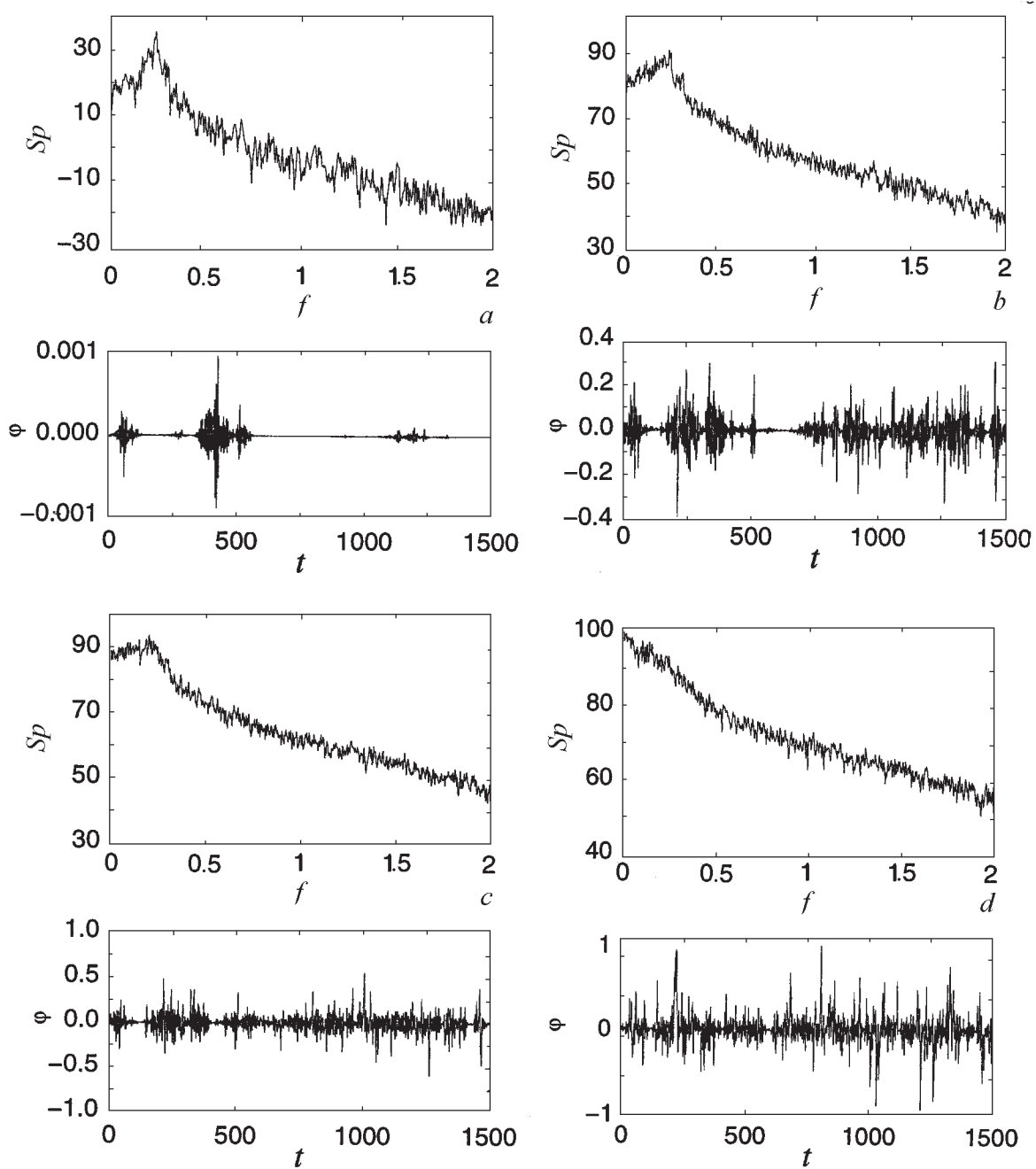

Fig. 7. The evolution of the shape of pendulum oscillations and their power spectra with increase of noise intensity for $\omega_{0}=1, \beta=0.1, \alpha=100$, (a) $\kappa\left(2 \omega_{0}\right) / \kappa_{c r}=1.01$, (b) $\kappa\left(2 \omega_{0}\right) / \kappa_{c r}=1.56$, (c) $\kappa\left(2 \omega_{0}\right) / \kappa_{c r}=2.44$ and $(d) \kappa\left(2 \omega_{0}\right) / \kappa_{c r}=6.25$. After [36].

example, in the case when the intensity of the random suspension axis vibration is close to its threshold value, the dependence of the intensity of pendulum oscillations on the frequency of the additional harmonic forcing is of a resonant character, very much like a jet subject to an acoustic force (cf. Figs. 8 and 9). Similarly, the additional forcing can be used to control the noise-induced pendulum oscillations. It can be included by substitution into Eq. (3.1) of $\xi+a \cos \omega_{a} t$ in place of $\xi$, where $a$ and $\omega_{a}$ are respectively the amplitude and frequency of the additional vibration applied to the suspension axis. If the frequency of the latter is relatively low, then this forcing intensifies the pendulum oscillations and lowers the excitation threshold; vice versa, a relatively high-frequency forcing suppresses the pendulum oscillations and increases the excitation threshold. The intensification of 


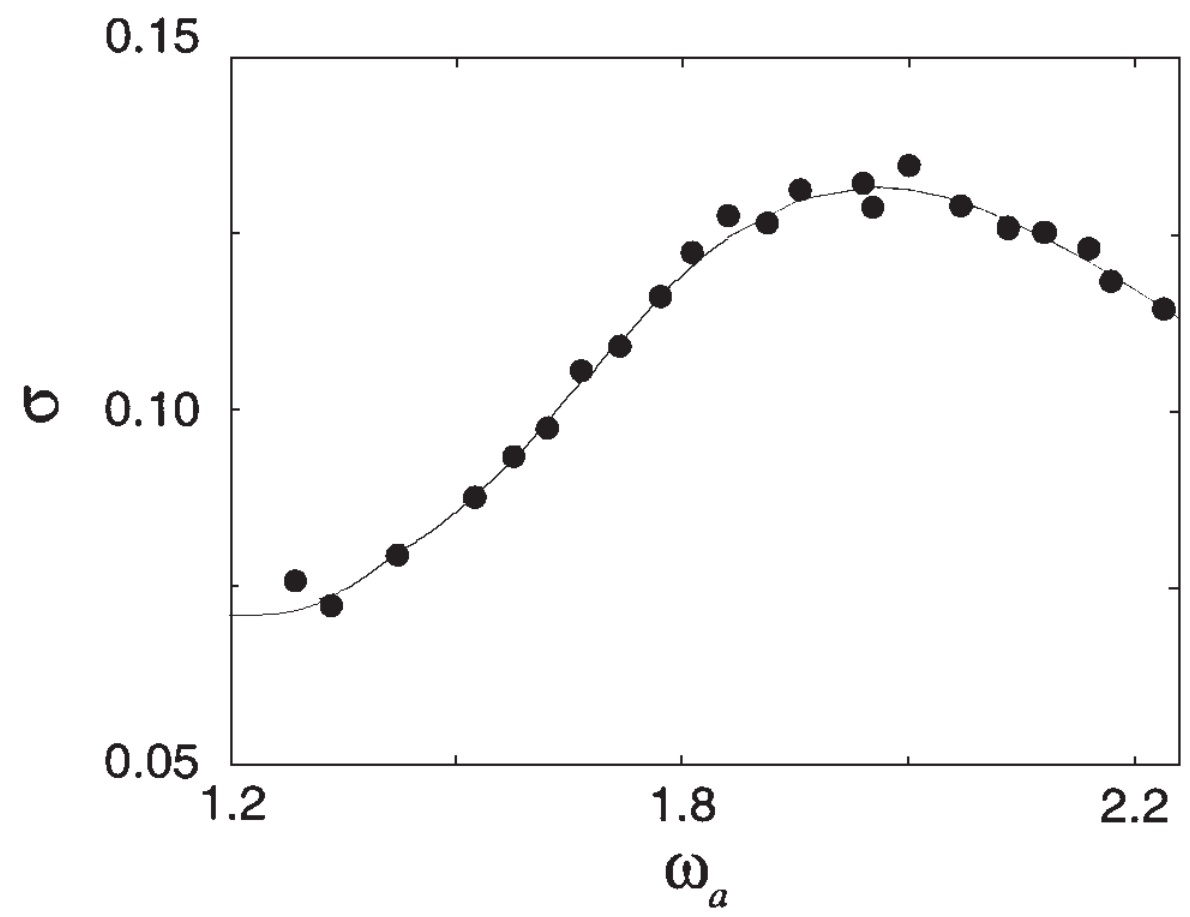

Fig. 8. The experimental dependences of $\epsilon_{u}=\sqrt{\overline{u^{2}}} / U_{0}$ on $\epsilon_{u \mathrm{a}}=\sqrt{\overline{\overline{u_{\mathrm{a}}^{2}}}} / U_{0}$ for $x / D$ and different values of the Strouhal number shown near the corresponding curves. After [36].

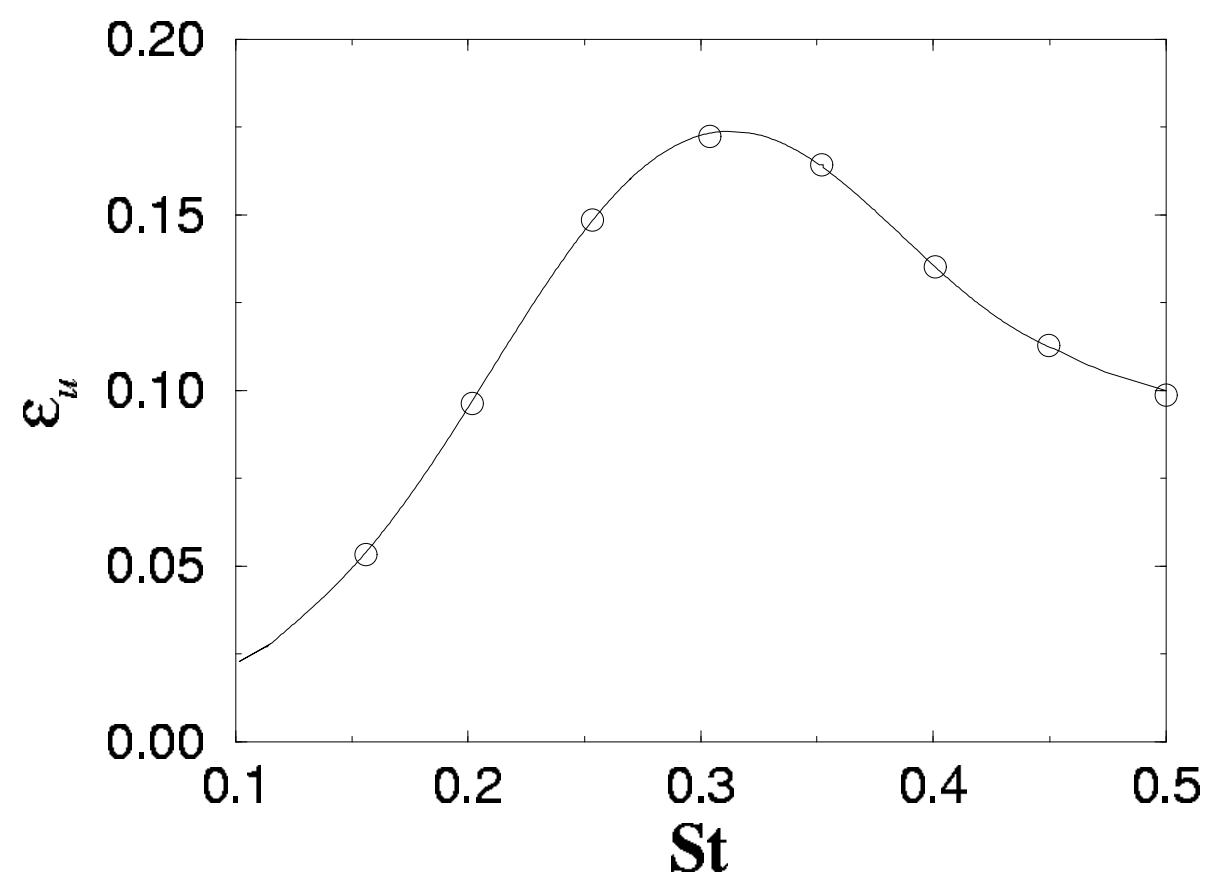

Fig. 9. The dependence of $\epsilon_{u}$ on St for $\epsilon_{u \text { a }}=0.02, x / D=4$ constructed from the data given in figure 8 . In the absence of an acoustical disturbance $\epsilon_{u} \approx 0.04$. After [36]. 

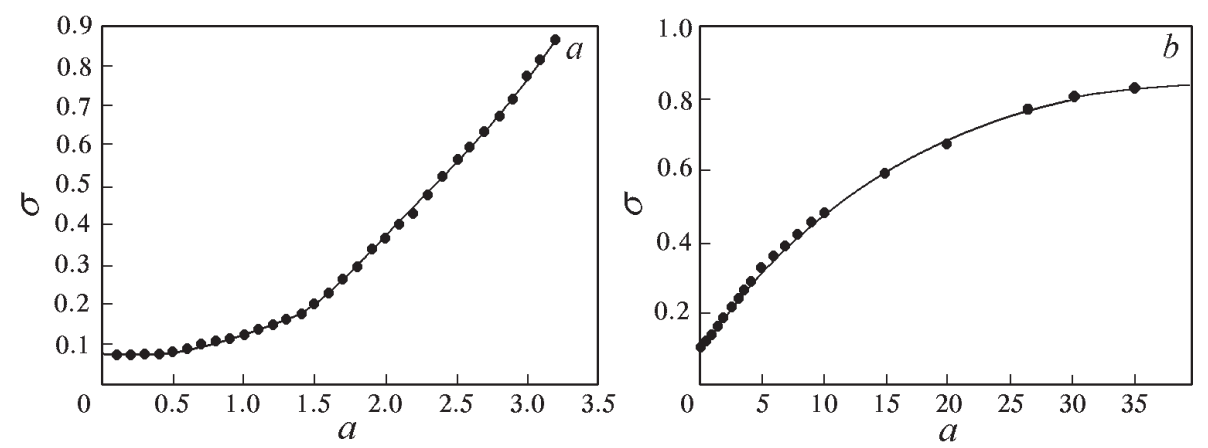

Fig. 10. The dependences of $\sigma$ on the amplitude $a$ of low-frequency oscillation for $\omega_{0}=1, \beta=0.1$, $\alpha=100,(a) \kappa\left(2 \omega_{0}\right) / \kappa_{c r}=1.89, \omega_{a}=0.3$ and $(b) \kappa\left(2 \omega_{0}\right) / \kappa_{c r}=2.23, \omega_{a}=1.5$. After [36].

the pendulum oscillations by a low-frequency additional vibration is illustrated in Fig. 10 for two values of the vibration frequency. We see that the lower the forcing frequency is, the larger the oscillation variance becomes. As in the case of jets [49], the pendulum's oscillation amplitude saturates at large forcing amplitude.

We now consider in detail the possibility of suppressing noise-induced pendulum oscillations by the addition of a vibration. Numerical simulation of Eq. (3.1) with $\xi+a \cos \omega_{a} t$ in place of $\xi$ as above, but with $\omega_{a}>2$, shows that such suppression can occur. The results of such simulations are presented in Figs. 11 and 12. It is evident from Fig. 11(a) that small amplitude vibrations have little or no effect on the noise-induced oscillations. As the amplitude increases, however, the intensity of the noise-induced oscillations decreases rapidly and the duration of the "laminar" phases correspondingly increases, as shown in Figs. $11 b-e$. When the amplitude exceeds a certain critical value (for $\omega_{a}=19.757$ it is equal to 42) the oscillations are suppressed entirely. As the amplitude increases further, the oscillations reappear, but this is because the conditions for parametric resonance come into play. For smaller frequencies $\omega_{a}$, the behavior of the pendulum oscillations is different. The dependences of the variance of the angle $\varphi$ on $a$ for a number of vibration frequencies are shown in Fig. 12. It is evident that the variance of $\varphi$ at first decreases, passes through a minimum, and then increases again. Note that this minimum value becomes smaller with increasing forcing frequency, but that it is attained for larger forcing amplitudes at higher frequencies. For sufficiently high frequencies the oscillations can be suppressed entirely (the case illustrated in Fig. 12 c)). The dependence shown in Fig. 12 a) closely resembles the corresponding dependence for a jet presented in Fig. 13. 

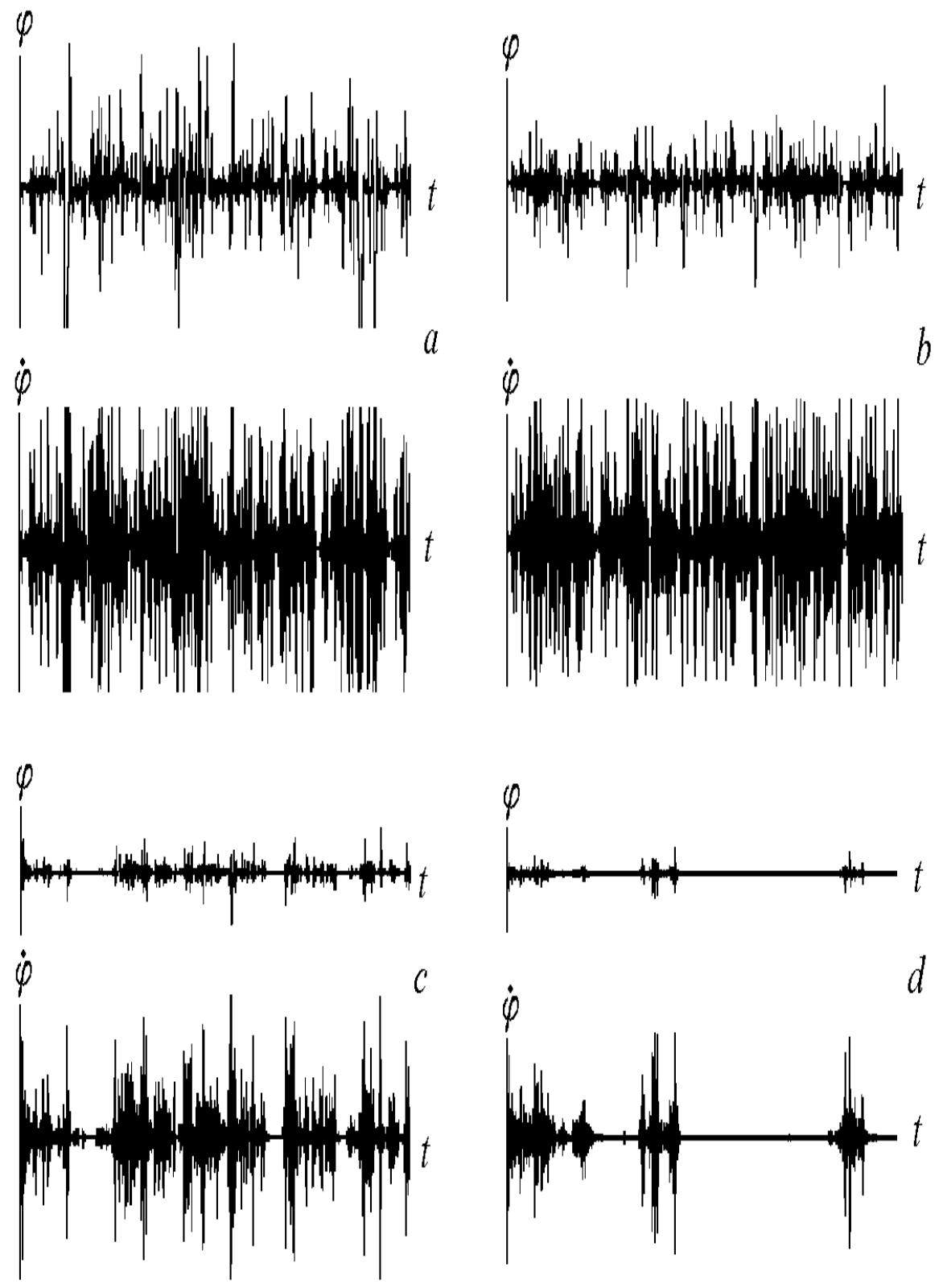

Fig. 11. The time evolutions of $\varphi(t)$ and $\dot{\varphi}(t)$ for $\omega_{0}=1, \beta=0.1, \alpha=100, \kappa\left(2 \omega_{0}\right) / \kappa_{c r}=5.6$, $\omega_{a}=19.757$ and: (a) $a=5$; (b) $a=15$; (c) $a=30$; and (d) $a=40$. After [26].

In contrast to the analysis in [36], where we considered the simplest case in which the acoustic forcing and noise are both multiplicative, we now allow for small additive constituents of the noise and acoustic wave. In this case the equation describing the oscillations of the corresponding pendulum model (with a randomly vibrated suspension axis and a periodic force) become

$\ddot{\varphi}+2 \beta\left(1-\alpha v_{1}^{2}\right) v_{1}+\omega_{0}^{2}\left(1+a_{\mathrm{m}} \cos \omega_{a} t+\xi_{\mathrm{m}}(t)\right) \sin \varphi=A \cos \omega_{a} t+\xi(t)$, 

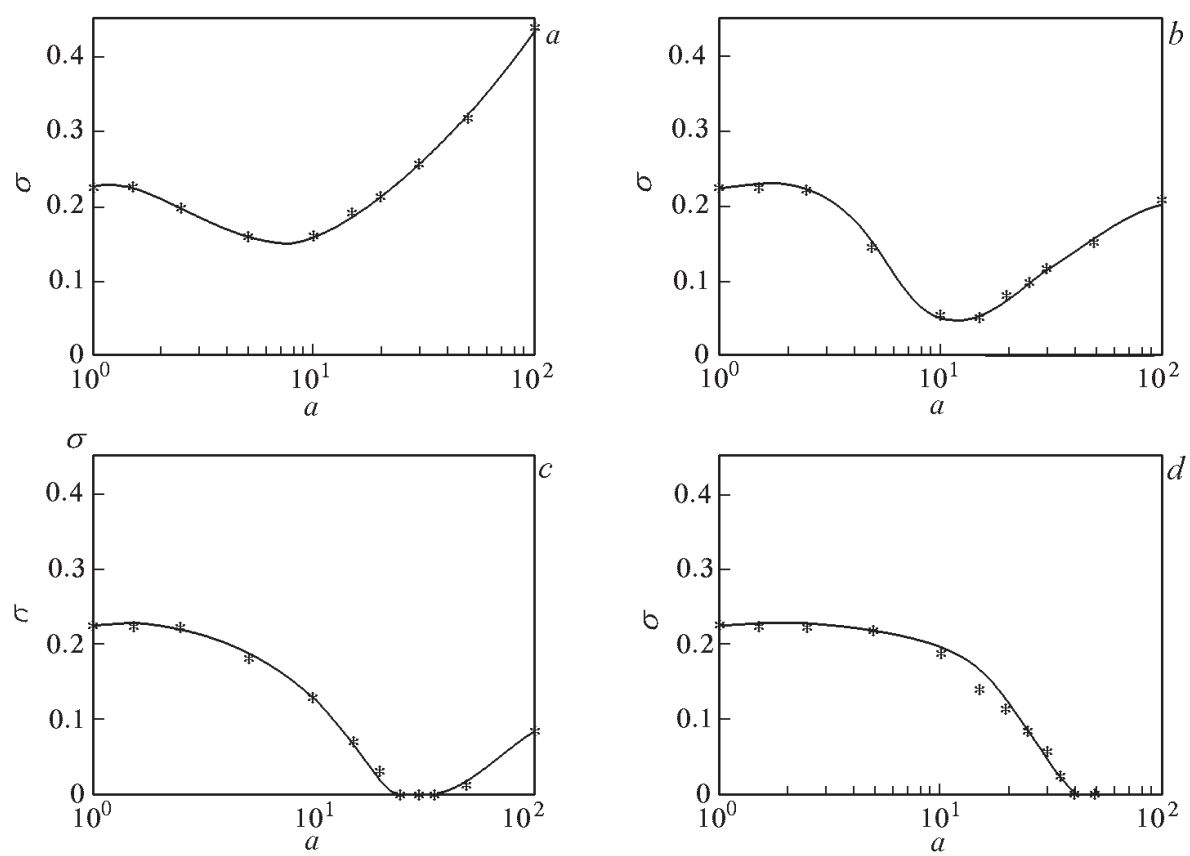

Fig. 12. The dependences of $\sigma$ on $a$ for $\omega_{0}=1, \beta=0.1, \alpha=100, \kappa\left(2 \omega_{0}\right) / \kappa_{c r}=5.6$ and: (a) $\omega_{a}=3.5 ;(b) \omega_{a}=6 ;(c) \omega_{a}=11 ;$ and $(d) \omega_{a}=19.757$. After [36].
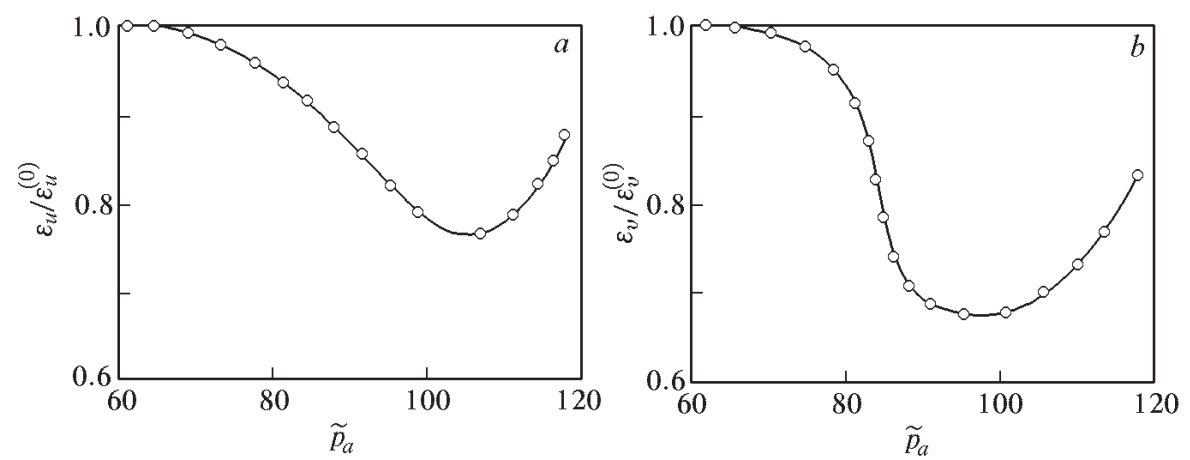

Fig. 13. The experimental dependences of the relative root-mean-square pulsation of the suppression factors $\epsilon_{u} / \epsilon_{u}^{(0)}$ and $\epsilon_{v} / \epsilon_{v}^{(0)}$ of the longitudinal and radial components of hydrodynamical velocity on the relative amplitude of acoustic pressure $\tilde{p}_{\mathrm{a}}$ measured in decibels, for $\mathrm{St}=2.35$, $x / D=8 ; \epsilon_{u}^{(0)}$ and $\epsilon_{v}^{(0)}$ are relative pulsations of the longitudinal and radial velocity components in the absence of acoustic forcing. After [36].

where $v_{1}=\dot{\varphi}, \xi_{\mathrm{m}}(t)$ and $\xi(t)$ are comparatively wide-band random processes, $\omega_{0}$ is the natural frequency of small pendulum oscillations, $a_{\mathrm{m}}$ and $A$ are the amplitudes of the multiplicative and additive constituents of the force, and $\omega_{a}$ is the external forcing frequency modelling the frequency of the acoustic wave. 
We further assume that $\omega_{0}^{2}$ is sufficiently small that Eq. (3.2) can be written as

$$
\dot{v}_{1}+2 \beta\left(1-\alpha v_{1}^{2}\right) v_{1}=A_{1} \cos \omega_{a 1} t+\xi_{1}(t)
$$

It should be noted that Eq. (3.3) is similar to Eq. (2.1). It can be reduced to Eq. (2.1) by setting $\alpha=1, \beta=-0.5, v_{1}=-v, A_{1}=-A, \xi_{1}(t)=-\xi(t)$. under these conditions Eq. (3.3 becomes

$$
\dot{v}+v^{3}-v=A \cos \omega_{a} t+\xi(t)
$$

Taking account (2.5) for the third moment, we split $v(t)$ into $s(t)$ and $n(t)$ as

$$
v(t)=s(t)+n(t)
$$

where $s(t)$ and $n(t)$ are described by the equations

$$
\begin{aligned}
& (1+b) \dot{s}+c s=A \cos \omega_{a} t, \\
& \dot{n}+n^{3}-n+\left(3 n^{2}-1-c\right) s-b \dot{s}=\xi(t),
\end{aligned}
$$

where

$$
c=3 m_{2}-1+a
$$

The Fokker-Planck equation corresponding to Eq. (3.7), in its linear approximation with respect to $s$, is

$\frac{\partial w(n, t)}{\partial t}-\frac{\partial}{\partial n}\left(2 \beta\left(n-\alpha\left(n^{3}+3 n^{2} s-c_{1} s-b \dot{s}\right) w(n, t)\right)=\frac{K}{2} \frac{\partial^{2} w(n, t)}{\partial n^{2}}\right.$.

As in section 1, it is convenient to seek a solution of Eq. (3.9) in the form of a sum

$$
w(n, t)=w_{0}(n)+\epsilon\left(w_{1}(n) s+w_{2}(n) \dot{s}\right)
$$

where functions $w_{0}(n), w_{1}(n)$ and $w_{2}(n)$ are described by the following equations:

$2 \beta\left(\alpha n^{3}-n\right) w_{0}(n)-\frac{K}{2} \frac{\partial w_{0}(n)}{\partial n}=0$ 
$-\omega_{a}^{2} w_{2}-2 \beta \frac{\partial}{\partial n}\left(\left(n-\alpha n^{3}\right) w_{1}(n)\right)-\frac{K}{2} \frac{\partial^{2} w_{1}}{\partial n^{2}}=2 \beta \frac{\partial}{\partial n}\left(\left(3 \alpha n^{2}-c_{1}\right) w_{0}(n)\right)$.

$w_{1}-2 \beta \frac{\partial}{\partial n}\left(\left(n-\alpha n^{3}\right) w_{2}(n)\right)-\frac{K}{2} \frac{\partial^{2} w_{2}}{\partial n^{2}}=-2 \beta \alpha b \frac{d w_{0}}{d n}$.

A solution of Eq. (3.11) vanishing for $n= \pm \infty$ is

$$
w_{0}(n)=C \exp \left[2 \beta\left(\frac{n^{2}}{K}-\alpha \frac{n^{4}}{4 K}\right)\right],
$$

where $C$ is the normalization constant.

From the condition $\langle n\rangle=0$, and the expression (2.5) for the first and third moments, we find the following relationships:

$$
\begin{aligned}
& \int_{-\infty}^{\infty} n w_{1}(n) d n=0, \quad \int_{-\infty}^{\infty} n w_{2}(n) d n=0, \\
& \int_{-\infty}^{\infty} n^{3} w_{1}(n) d n=c_{1}, \quad \int_{-\infty}^{\infty} n^{3} w_{2}(n) d n=b
\end{aligned}
$$

where $w_{1}(n)$ and $w_{2}(n)$ are solutions of Eqs. (3.12). It follows from (3.12) that these functions must be odd, i.e. they must satisfy tthe conditions $w_{1}(0)=w_{2}(0)=0$.

The general solution of the inhomogeneous equations (3.12) we can write as

$$
w_{1}(n)=w_{1 \mathrm{in}}(n)+w_{1 \mathrm{~h}}(n), \quad w_{2}(n)=w_{2 \mathrm{in}}(n)+w_{2 \mathrm{~h}}(n)
$$

where $w_{1 \text { in }}(n)$ and $w_{2 \text { in }}(n)$ are partial solutions of the inhomogeneous equations (3.14), which can be represented as sums of three components:

$$
\begin{aligned}
& w_{1 \text { in }}(n)=w_{11 \text { in }}(n)+w_{12 \text { in }}(n) c+w_{13 \text { in }}(n) b, \\
& w_{2 \text { in }}(n)=w_{21 \text { in }}(n)+w_{22 \text { in }}(n) c+w_{23 \text { in }}(n) b ;
\end{aligned}
$$

and $w_{1 \mathrm{~h}}(n), w_{2 \mathrm{~h}}(n)$ are partial solutions of the homogeneous parts of these equations, which may conveniently be represented as a linear combination of two independent partial 
solutions:

$$
w_{1 \mathrm{~h}}(h)=C_{1} w_{11 \mathrm{~h}}(n)+C_{2} w_{12 \mathrm{~h}}(n), \quad w_{2 \mathrm{~h}}(n)=C_{1} w_{21 \mathrm{~h}}(n)+C_{2} w_{22 \mathrm{~h}}(n),
$$

where $C_{1,2}$ are arbitrary constants.

Integrating Eqs. (3.14) over $n$ from 0 to $\infty$, subject to (3.15), (3.16) and (3.17), we obtain the following relationships:

$$
\begin{aligned}
& \int_{0}^{\infty} w_{11 \text { in }}(n) d n-\left.\frac{K}{2} \frac{d w_{21 \text { in }}(n)}{d n}\right|_{n=0}=0, \\
& \int_{0}^{\infty} w_{12 \text { in }}(n) d n-\left.\frac{K}{2} \frac{d w_{22 \text { in }}(n)}{d n}\right|_{n=0}=0, \\
& \int_{0}^{\infty} w_{13 \text { in }}(n) d n-\left.\frac{K}{2} \frac{d w_{23 \text { in }}(n)}{d n}\right|_{n=0}=-w_{0}(0), \\
& -\omega^{2} \int_{0}^{\infty} w_{21 \text { in }}(n) d n-\left.\frac{K}{2} \frac{d w_{11 \text { in }}(n)}{d n}\right|_{n=0}=-w_{0}(0), \\
& -\omega^{2} \int_{0}^{\infty} w_{22 \text { in }}(n) d n-\left.\frac{K}{2} \frac{d w_{12 \text { in }}(n)}{d n}\right|_{n=0}=-w_{0}(0), \\
& -\omega^{2} \int_{0}^{\infty} w_{23 \text { in }}(n) d n-\left.\frac{K}{2} \frac{d w_{13 \text { in }}(n)}{d n}\right|_{n=0}=0,
\end{aligned}
$$

where $w_{0}(0)=C(K)$.

Substituting (3.15), in view of (3.16), into Eqs. (3.14), we obtain from (3.14) the following equations for the unknowns $c_{1}, b, C_{1}$ and $C_{2}$ :

$$
\begin{aligned}
& \int_{-\infty}^{\infty} n\left(w_{11 \text { in }}(n)+w_{12 \text { in }}(n) c_{1}+w_{13 i n}(n) b+w_{11 \mathrm{~h}}(n) C_{1}+w_{12 \mathrm{~h}}(n) C_{2}\right) d n=0, \\
& \int_{-\infty}^{\infty} n\left(w_{21 \text { in }}(n)+w_{22 \text { in }}(n) c_{1}+w_{23 \operatorname{in}}(n) b+w_{21 \mathrm{~h}}(n) C_{1}+w_{22 \mathrm{~h}}(n) C_{2}\right) d n=0, \\
& \int_{-\infty}^{\infty} n^{3}\left(w_{11 \text { in }}(n)+w_{12 \mathrm{in}}(n) c_{1}+w_{13 \mathrm{in}}(n) b+w_{11 \mathrm{~h}}(n) C_{1}+w_{12 \mathrm{~h}}(n) C_{2}\right) d n=c_{1},
\end{aligned}
$$


$\int_{-\infty}^{\infty} n^{3}\left(w_{21 \text { in }}(n)+w_{22 \text { in }}(n) c_{1}+w_{23 \text { in }}(n) b+w_{21 \mathrm{~h}}(n) C_{1}+w_{22 \mathrm{~h}}(n) C_{2}\right) d n=b$.

It should be noted that the functions $w_{11 \text { in }}(n), w_{21 \text { in }}(n), w_{12 \text { in }}(n), w_{22 \text { in }}(n), w_{13 i n}(n)$, $w_{23 i n}(n), w_{11 \mathrm{~h}}(n), w_{21 \mathrm{~h}}(n), w_{12 \mathrm{~h}}(n)$, and $w_{22 \mathrm{~h}}(n)$ vanish for $n= \pm \infty$, though we have solved the Cauchy problem, but not the boundary value one.

It follows from our results that the effective values of $c, b$ and $a$ depend essentially on the signal frequency $\omega$ and on the noise intensity $K$.

Knowing $c, b, w_{11 \text { in }}(n), w_{12 \text { in }}(n), w_{13 \text { in }}(n), w_{21 \text { in }}(n), w_{22 \text { in }}(n), w_{23 \text { in }}(n), w_{11 \mathrm{~h}}(n), w_{12 \mathrm{~h}}(n)$, $w_{21 \mathrm{~h}}(n)$ and $w_{22 \mathrm{~h}}(n)$ we can use the formulæ (3.12) and (3.18) to calculate the complete probability distribution $w(n, t)$ and its change

$$
\Delta w(n, t)=w_{1}(n) s(t)+w_{2}(n) \dot{s}
$$

Subject to (3.18), we can rewrite (3.20) as

$$
\begin{aligned}
& \Delta w(n, t)=\left(w_{11 \text { in }}(n)+c w_{12 \text { in }}(n)+b w_{13 \text { in }}(n)+C_{1} w_{11 \mathrm{~h}}(n)+C_{2} w_{12 \mathrm{~h}}(n)\right) A \cos \omega t \\
& -\omega\left(w_{21 \text { in }}(n)+c w_{22 \text { in }}(n)+b w_{23 \text { in }}(n)+C_{1} w_{21 \mathrm{~h}}(n)+C_{2} w_{22 \mathrm{~h}}(n)\right) A \sin \omega t .
\end{aligned}
$$

As was to be expected, for $t=T$, the function $\Delta w(n, t)$ coincides with $\Delta w(n, 0)$.

\section{Brownian motion in fixed and rotating coordinate systems}

\subsection{Brownian motion in a fixed coordinate system}

Next, we consider the classical problem of a small sphere (Brownian particle) of radius $R$ and mass $m$ fluctuating in a fluid. The latter can be described by gas dynamics equations with random sources conditioned by its atomic/molecular structure [61]. Fluctuations of the thermodynamic variables (e.g. density, velocity, temperature) caused by these sources are called natural fluctuations because they are inherent and unremovable even in prin- 
ciple. In the mechanics of macroscopic continua these fluctuations are of course omitted. But a correct description of a Brownian particle's motion requires that they be taken explicitly into account. Klimontovich has shown [61] that this approach leads naturally to the well-known Langevin equation (see also [44,45]):

$$
\frac{d \mathbf{v}}{d t}+\gamma \mathbf{v}=\xi(t)
$$

where $\mathbf{v}$ is the Brownian particle's velocity, $\xi(t)$ is white noise with correlation function $\langle\xi(t) \xi(t+\tau)\rangle=K \delta(\tau)$,

$$
K=2 \gamma \frac{k T}{m},
$$

is half of the spectral density for the process $\xi(t), k$ is Boltzmann's constant, $T$ is the temperature of the medium,

$$
\gamma=\frac{6 \pi \eta R}{m}
$$

and $\eta$ is the kinematic viscosity. The noise $\xi(t)$ emerges on account of collisions between the Brownian particle and the molecules of the medium. The term $\gamma \mathbf{v}$ is the Stokes force which describes internal friction within the medium.

It follows from Eq. (4.1) that the spectral density of the Brownian particle's velocity is equal to

$$
S(\omega)=\frac{2 K}{\omega^{2}+\gamma^{2}}
$$

i.e. it is described by a Lorentzian lineshape of half-width $\gamma$. The correlation function for Brownian motion $C f(\tau)$ is an exponentially decreasing function defined by

$$
C f(\tau)=\frac{K}{2 \gamma} \exp (-\gamma|\tau|)
$$

In the one-dimensional case the Fokker-Planck equation corresponding to Eq. (4.1) $[44,45]$ is

$$
\frac{\partial w}{\partial t}+\frac{\partial G}{\partial v}=0
$$


where

$$
G=-\gamma v w-\frac{K}{2} \frac{\partial w}{\partial v}
$$

is the probability flow.

A stationary solution of Eq. (4.6) for the condition that the probability flow vanishes when $v \rightarrow \pm \infty$ is

$$
w(v)=C \exp \left(-\frac{\gamma v^{2}}{K}\right)
$$

It follows from this that the Brownian particle's velocity has a Gaussian probability distribution with normalization constant $C=\sqrt{\pi \gamma / K}$.

\subsection{Brownian motion in a rotating coordinate system}

We now consider a Brownian particle of mass $m$ moving near the Earth's surface with a translational velocity of $\mathbf{v}$ relative to Earth. First, we introduce a new coordinate system joint with the Brownian particle. This system rotates relative to Earth with angular velocity

$$
\Omega=\Omega_{1}+\Omega_{2}+\frac{\mathbf{v}}{R_{1}}+\frac{\mathbf{v}}{R_{2}}
$$

where $\Omega_{1}$ is the angular velocity at which the Earth rotates for $\mathbf{v}=0, R_{1}$ is the distance between the centre of the Earth and the Brownian particle, $\Omega_{2}$ is the orbital angular velocity of the Earth around the Sun for $\mathbf{v}=0$, and $R_{2}$ is the radius of the Earth's orbit which we assume to be circular.

The inertial forces acting on the Brownian particle are subdivided into centrifugal and Coriolis. The centrifugal force is

$$
f_{\mathrm{cf}}=-m\left\{\left(\Omega_{1}+\frac{\mathbf{v}}{R_{1}}\right)^{2} \mathbf{r}_{1}+\left(\Omega_{2}+\frac{\mathbf{v}}{R_{2}}\right)^{2} \mathbf{r}_{2}\right\}
$$


where $\mathbf{r}_{1}$ is the vector traced from the centre of the Earth to the Brownian particle, $\mathbf{r}_{2}$ is the vector traced from the Earth's axis of rotation around Sun to the Brownian particle.

It can readily be shown that

$$
\left|\mathbf{r}_{1,2}\right| \approx R_{1,2} \cos \varphi
$$

where $\varphi$ is the latitude at which the Brownian particle is situated.

Because the Brownian particle's linear velocity is relatively small, and the Earth's radius is large (so that $\left|\left(\mathbf{v} / R_{1}\right)^{2} \mathbf{r}_{1}\right| \ll\left|\Omega_{1}\right|$ ), we need take account only of the Coriolis force, which is

$$
f_{\text {Cor }}=2 m\left[\left(\boldsymbol{\Omega}_{1}+\boldsymbol{\Omega}_{2}\right) \mathbf{v}\right]
$$

The square brackets in (4.12) imply a vector product.

Further, we suggest that $\mathbf{v}$ is orthogonal to $\Omega_{1}$ and we take into account that $\left|\Omega_{2}\right| \ll\left|\Omega_{1}\right|$, in which case

$$
f_{\text {Cor }} \approx 2 m \Omega_{1} v
$$

Because the distances $R_{1,2}$ undergoes small changes with time at periods close to $T_{1} \approx$ $2 \pi / \Omega_{1}$ (day), and $T_{2} \approx 2 \pi / \Omega_{2}$ (year), the centrifugal and Coriolis forces must change with the same periods. In addition, because of the nonlinearity of the equations of motion, the velocities of the Brownian particles may contain combination frequencies ${ }^{2} \Omega_{3,4}=\Omega_{1} \pm \Omega_{2}$.

$\overline{2}$ Note that, in trying to account for his experimental data, Shnol' asserts [35] that one of the periods of the processes observed by him is equal to the sidereal day, which is shorter than the solar day by approximately 4 minutes. But it is difficult to explain how the sidereal day could influence the Brownian particle's velocity. It seems more probably that the existence of a period approximately equal to the sidereal day can be accounted for by the presence of a period corresponding to the combination frequency $\Omega_{3}=\Omega_{1}+\Omega_{2}$. The excitation of combination frequencies is to be expected given the nonlinearity of the Brownian particle's equations of motion. Because $T_{\text {sol }}=2 \pi / \Omega_{1}=1440$ minutes (solar day), $T_{\exp } \approx 1436$ minutes (the period 
Taking account of (4.13), the approximate equation of motion for a Brownian particle is

$$
\frac{d v}{d t}+\gamma v+\left(\Omega_{1}-\frac{v}{R_{1}}\right)^{2}\left|r_{1}\right|+\left(\Omega_{2}-\frac{v}{R_{2}}\right)^{2}\left|r_{2}\right|-2 \Omega_{1} v=\xi(t)
$$

where $\left|r_{1,2}\right|$ are defined by (4.11).

The Fokker-Planck equation for the probability density $w(v)$ corresponding to Eq. (4.14) is

$$
\frac{\partial w}{\partial t}+\frac{\partial G(v)}{\partial v}=0
$$

where

$$
G(v)=-\gamma v-\left\{\left(\Omega_{1}-\frac{v}{R_{1}}\right)^{2}\left|r_{1}\right|+\left(\Omega_{2}-\frac{v}{R_{2}}\right)^{2}\left|r_{2}\right|-2 \Omega_{1} v\right\} w(v)-\frac{K}{2} \frac{\partial w}{\partial v}
$$

The stationary solution of Eq. (4.15) under the condition $G(v) \equiv 0$ is defined by the boundary-value problem described by the equation

$$
\frac{d w}{d v}-\frac{2 \gamma v}{K}+\frac{2}{K}\left\{\left(\Omega_{1}-\frac{v}{R_{1}}\right)^{2}\left|r_{1}\right|+\left(\Omega_{2}-\frac{v}{R_{2}}\right)^{2}\left|r_{2}\right|-2 \Omega_{1} v\right\} w(v)=0
$$

with boundary conditions $w( \pm \infty)=0$. Solving this boundary-value problem we find

$$
\begin{aligned}
w(v)= & C \exp \left\{-\frac{\gamma v^{2}}{K}-\frac{2 v}{K}\left[\left|r_{1}\right|\left(\Omega_{1}^{2}-2 \frac{\Omega_{1} v}{R_{1}}+\frac{v^{2}}{3 R_{1}^{2}}\right)\right.\right. \\
& \left.\left.+\left|r_{2}\right|\left(\Omega_{2}^{2}-2 \frac{\Omega_{2} v}{R_{2}}+\frac{v^{2}}{3 R_{2}^{2}}\right)\right]-\Omega_{1} v^{2}\right\} .
\end{aligned}
$$

We see from this that the probability distribution differs in many respects from a normal (Gaussian) one. First, it depends on the sign of the velocity $v$. For $v>0$ it is a nonmonotonic function of $v$ that can have several extrema but, for $v<0$, it is a monotonically decreasing function. It is clear from (4.18) that the model considered above can contain

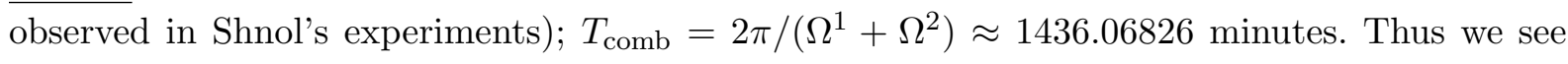
that the period $T_{\text {exp }}$ observed in Shnol's experiments is very close to $T_{\text {comb. It seems unlikely }}$ that this is a coincidence. So we believe that, in reality, the sidereal day plays no part in Shnol's experiments. 
one, two or three extrema in the probability distribution depending on the number of real roots of the cubic equation

$$
-\frac{\gamma v^{2}}{K}-\frac{2 v}{K}\left[r_{1}\left(\Omega_{1}^{2}-2 \frac{\Omega_{1} v}{R_{1}}+\frac{v^{2}}{3 R_{1}^{2}}\right)+r_{2}\left(\Omega_{2}^{2}-2 \frac{\Omega_{2} v}{R_{2}}+\frac{v^{2}}{3 R_{2}^{2}}\right)\right]-\Omega_{1} v^{2}=0 .
$$

These results correspond qualitatively to Shnol's experimental observations. Noting that $R_{1,2}$ and $r_{1,2}$ depend slightly on the local time, we conclude that the form of the probability distribution also depends on the local time, which is again in agreement with Shnol's experimental results.

To conclude this section, we note that the results obtained above are similar to those for the interaction between Brownian and hypothetical cosmic particles [40], consistent with Shnol's assumption that the phenomena he observed are of a universal character [35].

\section{Alpha decay in stationary and rotating coordinate systems}

\subsection{Alpha decay in a stationary coordinate system (classical model)}

Shnol' has provided experimental evidence [35] that a wide range of terrestrial random processes is correlated with astrophysical influences, e.g. the Earth's rotation. Other researchers $[32,33]$ have shown experimentally that the rates of some such processes correlate with the distance from Earth to Sun. Based on multiple experiments, Shnol' arrived at the conclusion that the behavior of all such processes is universal. We use this as a justification for analysing a classical model of alpha emission. The latter is, of course, considerably simpler than the quantum mechanical model needed to give an accurate description: in reality, alpha decay involves quantum tunnelling of the particle through the potential barrier formed by the sum of Coulomb and nuclear forces $[62,63]$. In contrast, the approach we adopt here involves analysis of the known classical model of noise-induced passage of a particle over a potential barrier, as developed by Pontryagin, Andronov and Witt in 1933 [64,65] and subsequently broadened and developed by Stratonovich and Landa [24]. The advantage of doing so is that it enables us to calculate analytically the probability density for the alpha decay rate and thus to determine the effect exerted on it by the Earth's 
rotation. Note that the alpha decay problem differs mathematically from the Brownian motion problem, to a considerable extent. The main difference is that escape of an alpha particle from the nucleus consists simply of its transition through the potential barrier, because the alpha particle energy is $5-10 \mathrm{eV}$, whereas the height of the Coulomb potential barrier for heavy nuclei is $25-30 \mathrm{eV}[62,63]$; the motion of Brownian particles, on the other hand, is caused by continuous collisions with the molecules of the liquid in which they are situated.

Supposing that the motion of an alpha particle to the nuclear boundary is similar to the motion of a lightweight classical particle in a viscous medium, we write a model equation as

$$
m \ddot{r}+\gamma \dot{r}+\frac{d V(r)}{d r}=\xi(t)
$$

where $r$ is the distance between the centres of the alpha particle and the nucleus, $\dot{r} \equiv v$ is the alpha particle velocity, $V(r)$ is a sum of nuclear, Coulomb and centrifugal potentials, $\xi(t)$ is white noise of intensity $K=\langle\xi(t) \xi(t+\tau)\rangle$, and $\gamma \dot{r}$ is the effective dissipative force akin to the Stokes force for a Brownian particle [40]. Further, we will assume that the mass of alpha particle is so small that the condition

$$
m \ddot{r} \ll \gamma \dot{r} .
$$

is fulfilled.

On the assumption that the alpha particle mass is much less than that of the nucleus, the potential $V(r)$ can be described $[62,63]$ by

$$
V(r)=V_{\text {nucl }}+\frac{Z e^{2}}{r}-\frac{\hbar^{2}}{2 m} \frac{L(L+1)}{r^{2}}
$$

where $V$ is the nuclear potential, $Z$ is the number of protons in the nucleus, $e$ is the electronic charge, $\hbar$ is Planck's constant, and $L$ is the alpha particle's orbital angular momentum. It follows from (5.3) that a potential well exists inside the nucleus. Its form can be calculated if the dependence of the nuclear potential on $r$ is known. In Gamow's 1927 theory of alpha decay, based on quantum mechanical tunnelling, the potential well 

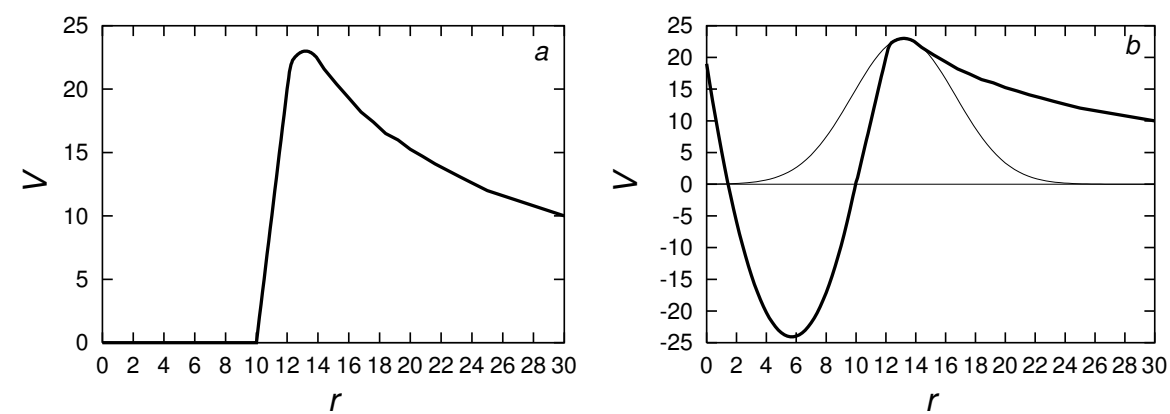

Fig. 14. (a) An example of the dependence $V(r)$ for the nucleus ${ }^{230}$ Th where $r$ is the distance from the nuclear center in Fermi-units, taken from [62]. (b) The same dependence but approximating the potential inside the well by a parabola (bold line).

was assumed to be rectangular.

For our purposes, however, it is more convenient to approximate the potential by a parabola whose parameters may be chosen such that, outside the well, the potential takes the form shown in Fig. $14(a)$. In so doing, the potential inside the well can be approximated by the parabola:

$$
V(r)=V_{0}\left(a_{1}-\frac{a_{2} r}{r_{m}}+\frac{a_{3} r^{2}}{r_{m}^{2}}\right) \equiv V_{0}\left(\frac{a_{3}\left(r-r_{\min }\right)^{2}}{r_{m}^{2}}-b\right),
$$

where $a_{1}=0.826, a_{2}=6.5666, a_{3}=5.7549, b=a_{2}^{2} /\left(4 a_{3}\right)-a_{1} \approx 1.0472, r_{m} \approx 10 F$ is the well width, $r_{\min }=a_{2} r_{m} /\left(2 a_{3}\right)$ is the value of $r$ for which the potential is minimal, and $V_{0} \approx 23$ is the maximum value of the potential. As in Fig. 14(a), we express the distance from the nuclear center in Fermi units ${ }^{3}$. Subject to (5.4) the dependence of the potential $V$ on distance $r$ takes the form shown in Fig. 14(b). The minimum of the potential corresponds to a stable equilibrium for the alpha particle, and the maximum to an unstable one $\left(r=r_{0} \approx 14.2 F\right)$. Escape of the alpha-particle occurs when it surmounts the potential barrier, i.e. when $r$ becomes equal to $r_{0}$. So the problem of escape reduces to the well-known problem of the achievement of a boundary $[44,45]$.

The potential $V(r)$ shown in Fig. $14 b$ for $1 \leq r / r_{m} \leq r_{0} / r_{m}$, can be approximated by the polynomial

$\overline{3 \text { Fermi }}=10^{-15} \mathrm{~m}$. 


$$
\begin{aligned}
V(r)= & V_{0}\left(\frac{r}{r_{m}}-1\right)\left[5.5187-40.2072\left(\frac{r}{r_{m}}-1\right)+424.36\left(\frac{r}{r_{m}}-1\right)^{2}-\right. \\
& \left.-1690.1348\left(\frac{r}{r_{m}}-1\right)^{3}+2136.4739\left(\frac{r}{r_{m}}-1\right)^{4}\right]
\end{aligned}
$$

where $V_{0}=23$.

We note that, near the maximum. $V(r)$ is well approximated by the Gaussian curve

$$
V(r) \approx V_{0} \exp \left[-k_{1}\left(1-\frac{r}{r_{0}}\right)^{2}\right],
$$

where $k_{1}=7.26$. This curve is indicated by the thin line in Fig. $14(b)$.

Under the condition (5.2), Eq. (5.1) becomes

$$
\gamma \dot{r}+\frac{d V(r)}{d r}=\xi(t)
$$

Corresponding to Eq. (5.7) we have the Fokker-Planck equation

$$
\frac{\partial w(r)}{\partial t}=\frac{1}{\gamma} \frac{\partial}{\partial r}\left(\frac{d V(r)}{d r} w(r)\right)+\frac{K}{2 \gamma^{2}} \frac{\partial^{2} w(r)}{\partial r^{2}} .
$$

Its stationary solution is

$$
w_{0}(r)=C \exp \left(-\frac{2 \gamma V(r)}{K}\right)
$$

where $C$ is the normalization constant. As expected, the probability has a maximum at the point $r=r_{\min }$ and a minimum at $r=r_{0}$.

Substituting (5.4) into (5.9) and introducing a dimensionless parameter,

$$
a=\frac{2 \gamma V_{0}}{K}
$$

we find the probability distribution inside the potential well in the form:

$$
w_{0}(r)=C_{1} \exp \left[-\left(\frac{a a_{3}\left(r-r_{\min }\right)^{2}}{r_{m}^{2}}\right)\right],
$$

where $C_{1}=C e^{a b}$. 
It follows from (5.11) that we have a Gaussian probability distribution inside the potential well, bounded from both sides, with a mean value $r_{\text {min }}$ and a variance $\sigma=r_{m} / \sqrt{a a_{3}}$. If $\sigma \ll r_{\min }$ then, in calculating $C_{1}$, we can integrate $w_{0}(r)$ over $r$ approximately, from $-\infty$ to $\infty$, yielding

$$
C_{1} \approx r_{m} \sqrt{\frac{a a_{3}}{\pi}}
$$

Let the distance between the alpha particle and the nuclear center be $r$ at the initial moment of time. The probability $P(\tau, r)$ that the coordinate $r$ does not reach the boundary value $r_{0}$ during the time interval $\tau$ is associated with the probability density $w\left(r^{\prime}, r, \tau\right)$ of the representative points in phase space not touching the boundary $r_{0}$ during the time $\tau$, which is determined by the relationship [24]:

$$
P(\tau, r)=\int_{0}^{r_{0}} w\left(r^{\prime}, r, \tau\right) d r^{\prime}
$$

where the distribution of density $w\left(r^{\prime}, r, \tau\right)$ is described by the equation

$$
\frac{\partial w\left(r^{\prime}, r, \tau\right)}{\partial \tau}=\frac{\partial}{\partial r^{\prime}}\left(\frac{1}{\gamma} \frac{d V\left(r^{\prime}\right)}{d r^{\prime}} w\left(r^{\prime}, r, \tau\right)\right)+\frac{K}{2 \gamma^{2}} \frac{\partial^{2} w\left(r^{\prime}, r, \tau\right)}{\partial\left(r^{\prime}\right)^{2}}
$$

with boundary condition

$$
\left.w\left(r^{\prime}, r, \tau\right)\right|_{r^{\prime}=r_{0}}=0
$$

and initial condition

$$
w\left(r^{\prime}, r, 0\right)=\delta\left(r^{\prime}-r\right)
$$

The zero boundary condition for the function $w\left(r^{\prime}, r, \tau\right)$ is associated with the fact that, at $\tau>0$, there are practically no trajectories near the boundary, and it is impossible for any points to reach the boundary which have not yet touched the boundary. But it is just these trajectories that are described by the probability density $w\left(r^{\prime}, r, \tau\right)$.

Knowing the probability $P(\tau, r)$ we can calculate the expectation of the boundary achieve- 
ment time $\langle\tau(r)\rangle$ as

$$
\langle\tau(r)\rangle=-\int_{0}^{\infty} \tau \frac{\partial P(\tau, r)}{\partial \tau} d \tau=-\left.\tau P(\tau, r)\right|_{0} ^{\infty}+\int_{0}^{\infty} P(\tau, r) d \tau
$$

Given that $P(0, r)=1, P(\infty, r)=0$, and $\lim _{\tau \rightarrow \infty} \tau P(\tau, r)=0$, we find from (5.17) that

$$
\langle\tau(r)\rangle=\int_{0}^{\infty} P(\tau, r) d \tau
$$

Because the probability $P(\tau, r)$ depends on the value of $r$ at the initial instant of time, the expectation $\langle\tau\rangle$ has must also depend on $r$. To derive equations for $P(\tau, r)$ and $\langle\tau(r)\rangle$, we will consider them as functions of $r$. First, we note that the probability density $w\left(r^{\prime}, r, \tau\right)$ as a function of variables $r$ and $\tau$ satisfies an equation conjugate with Eq. (5.14), namely

$$
\frac{\partial w\left(r^{\prime}, r, \tau\right)}{\partial \tau}=-\frac{1}{\gamma} \frac{d V(r)}{d r} \frac{\partial w\left(r^{\prime}, r, \tau\right)}{\partial r}+\frac{K}{2 \gamma^{2}} \frac{\partial^{2} w\left(r^{\prime}, r, \tau\right)}{\partial r^{2}}
$$

This equation (5.19) is called the first Kolmogorov equation [66].

Integrating Eq. (5.19) over $r^{\prime}$ for $r_{\min } \leq r^{\prime} \leq r_{0}$ and taking account of (5.13), we find the equation for the probability $P(\tau, r)$ :

$$
\frac{\partial P(\tau, r)}{\partial \tau}=-\frac{1}{\gamma} \frac{d V(r)}{d r} \frac{\partial P(\tau, r)}{\partial r}+\frac{K}{2 \gamma^{2}} \frac{\partial^{2} P(\tau, r)}{\partial r^{2}}
$$

Let us present $\partial P(\tau, r) / \partial \tau$ as the Fourier transform of the characteristic function $\Theta(i \nu, r)$ :

$$
\frac{\partial P(\tau, r)}{\partial \tau}=\frac{1}{\pi} \int_{-\infty}^{\infty} \Theta(i \nu, r) \exp (-i \nu \tau) d \nu
$$

The inverse transformation is

$$
\Theta(i \nu, r)=-\int_{0}^{\infty} \frac{\partial P(\tau, r)}{\partial \tau} \exp (i \nu \tau) d \tau
$$

We expand the right-hand side of (5.22) as a series in $i \nu$ in the vicinity of $i \nu=0$ :

$$
\Theta(i \nu, r)=\sum_{k=0}^{\infty} \frac{(i \nu)^{k}}{k !} m_{k}(r)
$$


where $m_{k}(r)=-\int_{0}^{\infty} \tau^{k} \frac{\partial P(\tau, r)}{\partial \tau} d \tau$ is the $k$-th moment of the boundary achievement time. Since $P(\infty, r)=0$ and $P(0, r)=1$, it follows that $m_{0}(r)=P(0, r)-P(\infty, r)=1$.

Differentiating both parts of Eq. (5.20) with respect to $\tau$, multiplying by $\exp (i \nu \tau)$ and integrating over $\tau$ from 0 to $\infty$, we obtain the following equation for the characteristic function $\Theta(i \nu, E, r)$ :

$$
-i \nu \Theta(i \nu, r)=-\frac{1}{\gamma} \frac{d V(r)}{d r} \frac{\partial \Theta(i \nu, r)}{\partial r}+\frac{K}{2 \gamma^{2}} \frac{\partial^{2} \Theta(i \nu, r)}{\partial r^{2}}
$$

Substituting (5.23) into Eq. (5.24) and equating coefficients of the same power exponents of $i \nu$, we can find equations for all moments of the boundary achievement time. In particular, for the mathematical expectation of the boundary achievement time $M(r)=m_{1}(r)$ we obtain:

$$
-\frac{1}{\gamma} \frac{d V(r)}{d r} \frac{\partial M(r)}{\partial r}+\frac{K}{2 \gamma^{2}} \frac{\partial^{2} M(r)}{\partial r^{2}}+1=0
$$

Equations of the forms (5.21) and (5.25), first derived by the Russian mathematician Pontryagin [64] are often called the first and second Pontryagin equations respectively.

To solve Eq. (5.25) we must set two boundary conditions. One of these is immediately evident. It is

$$
M\left(r_{0}\right)=0
$$

For the second boundary condition we set the requirement that the mean time for reaching the boundary should be maximum for $r=r_{\min }$ :

$$
\left.\frac{d M(r)}{d r}\right|_{r=r_{\min }}=0
$$

A solution of Eq. (5.25) obeying these boundary conditions (5.26) and (5.27) is

$$
M(r)=\frac{2 \gamma^{2}}{K} \int_{r}^{r_{0}} \exp \left(\frac{2 \gamma}{K} V(z)\right) \int_{r_{\min }}^{z} \exp \left(-\frac{2 \gamma}{K} V(x)\right) d x d z
$$


This expression (5.28) can be simplified much as was done earlier [65,24]. We take into account that the integrand in the inner integral has its greatest value for $x=r_{\min }$, when $V(x)=V_{\min }$. That is why we can use formula (5.4) for calculating it. In so doing the inner integral is approximately equal to

$I_{1}(z) \approx \int_{r_{\min }}^{z} \exp \left[-a\left(\frac{a_{3}}{r_{m}^{2}}\left(x-r_{\min }\right)^{2}-b\right)\right] d x=\frac{r_{m} e^{a b}}{2} \sqrt{\frac{\pi}{a a_{3}}} \Phi\left(\sqrt{\frac{a a_{3}}{2}} \frac{z-r_{\min }}{r_{m}}\right)$,

where $\Phi(x)=\sqrt{\frac{2}{\pi}} \int_{0}^{x} \exp \left(-\frac{y^{2}}{2}\right) d y$ is the probability integral.

The integrand in the external integral (5.28) has its greatest value for $z=r_{0}$. Hence in calculating it we can use the expression (5.6), expanding the exponent into a series in $1-r / r_{0}$, i.e. we can set

$$
V(z) \approx V_{0}\left[1-k_{1}\left(1-\frac{z}{r_{0}}\right)^{2}\right]
$$

The inner integral $I_{1}(z)$ may be calculated approximately at the point $z=r_{\min }$, yielding

$$
M(r) \approx \gamma \frac{\pi r_{0} r_{m}}{\sqrt{a_{3} k_{1}}} \Phi\left[\sqrt{\frac{a k_{1}}{2}}\left(1-\frac{r}{r_{0}}\right)\right] \Phi\left(\sqrt{\frac{a a_{3}}{2}} \frac{r_{0}-r_{\min }}{r_{m}}\right) \exp ((b+1) a) .
$$

We note that, as expected, the exponent $(b+1) a$ in $(5.31)$ is proportional to the difference between the potentials at the positions of the unstable and stable equilibria.

Allowing for the fact that, on touching the boundary in phase space, the representative point can with equal probability either cross the boundary or return [45], we obtain the following approximate expression for the mean time $\tau$ of the transition through the boundary:

$$
\tau(r) \approx \frac{M(r)}{2} \approx \gamma \frac{\pi r_{0} r_{m}}{2 \sqrt{a_{3} k_{1}}} \Phi\left[\sqrt{\frac{a k_{1}}{2}}\left(1-\frac{r}{r_{0}}\right)\right] \Phi\left(\sqrt{\frac{a a_{3}}{2}} \rho\left(r_{0}\right)\right) \exp ((b+1) a) .
$$

The mean number of alpha particles radiated per unit time $(N(r))$ is inversely proportional to $\tau(r)$, i.e.

$$
N(r) \approx \frac{2 \sqrt{a_{3} k_{1}}}{\pi r_{0} r_{m} \gamma} \Phi^{-1}\left[\sqrt{\frac{a k_{1}}{2}}\left(1-\frac{r}{r_{0}}\right)\right] \Phi^{-1}\left(\sqrt{\frac{a a_{3}}{2}} \rho\left(r_{0}\right)\right) \exp (-(b+1) a) .
$$


We will call the function $N(r)$ the rate of alpha decay.

The expression (5.33) may conveniently be rewritten in terms of a dimensionless parameter

$$
N_{0}=\frac{2 \sqrt{a_{3} k_{1}}}{\pi r_{0} r_{m} \gamma} \Phi^{-1}\left[\sqrt{\frac{a k_{1}}{2}}\left(1-\frac{r_{\text {min }}}{r_{0}}\right)\right] \Phi^{-1}\left(\sqrt{\frac{a a_{3}}{2}} \frac{r_{0}-r_{\min }}{r_{m}}\right) \exp (-(b+1) a),
$$

where $N_{0}$ is the value of $N(r)$ for the initial value of $r$ equal to $r_{\min }$.

In so doing we obtain

$$
\frac{N}{N_{0}} \equiv n=\Phi\left[\sqrt{\frac{a k_{1}}{2}}\left(1-\frac{r_{\min }}{r_{0}}\right)\right] \Phi^{-1}\left[\sqrt{\frac{a k_{1}}{2}}\left(1-\frac{r}{r_{0}}\right)\right] .
$$

It is evident that only the integer parts of $N(r)$ and $N_{0}$ have physical meaning.

Because the initial value of the radius $r$, resulted in the radiation of alpha-particle, changes in the range $0 \leq r \leq r_{0}$, the relative rate of alpha decay $n(r)=N(r) / N_{0}$ can vary within the range from $n_{\min }$ to $\infty$, where

$$
n_{\min }=\Phi\left[\sqrt{\frac{a k_{1}}{2}}\left(1-\frac{r_{\min }}{r_{0}}\right)\right] \Phi^{-1}\left(\sqrt{\frac{a k_{1}}{2}}\right) .
$$

It is clear that the minimal rate of alpha decay depends on the parameter $a$ : it increases slowly with increasing $a$. As the value of the initial radius tends towards $r_{0}$, the rate of alpha decay tends to infinity but does so more slowly for larger $a$. The dependence of $n_{\min }=N_{\min } / N_{0}$ on $a$ is illustrated in Fig. 15 . We see that with increasing $a$ the value of $n_{\min }$ tends to 1.

Since the value of $r$ is random, with a probability density $w(r)$ defined by Eq. (5.8), the rate of alpha decay is also a random quantity with probability density $w(n)$. To calculate $w(n)$ we take account of the fact that

$$
w(r)=w(n) \frac{d n}{d r}
$$

which takes account of the probability density normalization, i.e. the equality $w(r) d r=$ $w(n) d n$. If this normalization is ignored, then we can set $w(n)=\left.w(r)\right|_{r=\phi(n)}$, where $\phi(n)$ is an inverse function. 


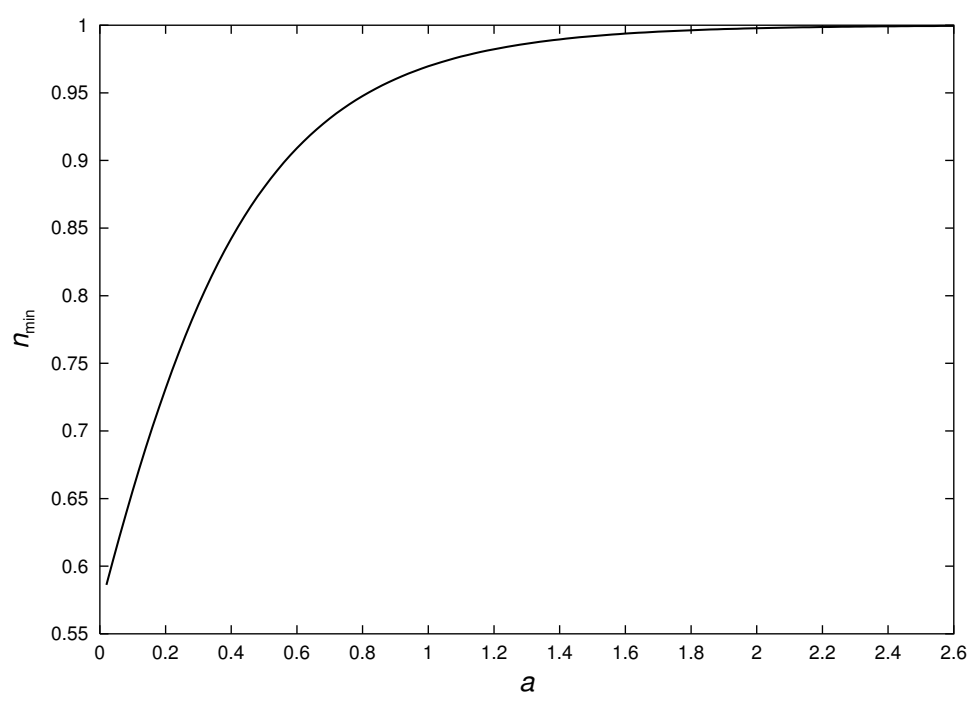

Fig. 15. The dependence on $a$ of $n_{\min }=N_{\min } / N_{0}$.

The derivative $d n / d r$ should be calculated as a function of $n$. For this it is sufficient to know the inverse function $\phi(n)$. The derivative $d n / d r$ can be expressed via $d \phi / d n$ as

$$
\frac{d n}{d r}=\frac{1}{\phi^{\prime}(n)}
$$

where $\phi^{\prime}(n)=d \phi / d n$.

To calculate the inverse function $\phi(n)$ it is necessary to solve Eq. (5.38) in terms of $r$. To perform this analytically is seemingly impossible. However, from formula (5.38) we can plot the dependences $\phi(n)$ for different values of parameter $a$. Examples of such plots for $a=0.5, a=2$ and $a=5$ are given in Fig. 16. We see that the function $\phi(n)$ changes sharply near $n_{\min }$.

Substituting (5.9) into (5.37) and taking account of (5.38 we obtain

$$
w(n)=C \exp \left(-\frac{a V(\phi(n))}{V_{0}}\right) \phi^{\prime}(n)
$$

In the neighborhood of the potential well, where the approximation (5.4) is valid, we have

$$
w(n)=C \exp \left[-a\left(a_{1}-\frac{a_{2} \phi(n)}{r_{m}}+\frac{a_{3} \phi^{2}(n)}{r_{m}^{2}}\right)\right] \phi^{\prime}(n) .
$$

The function $w(n)$ described by (5.40) decreases monotonically with increasing $n$, whereas 


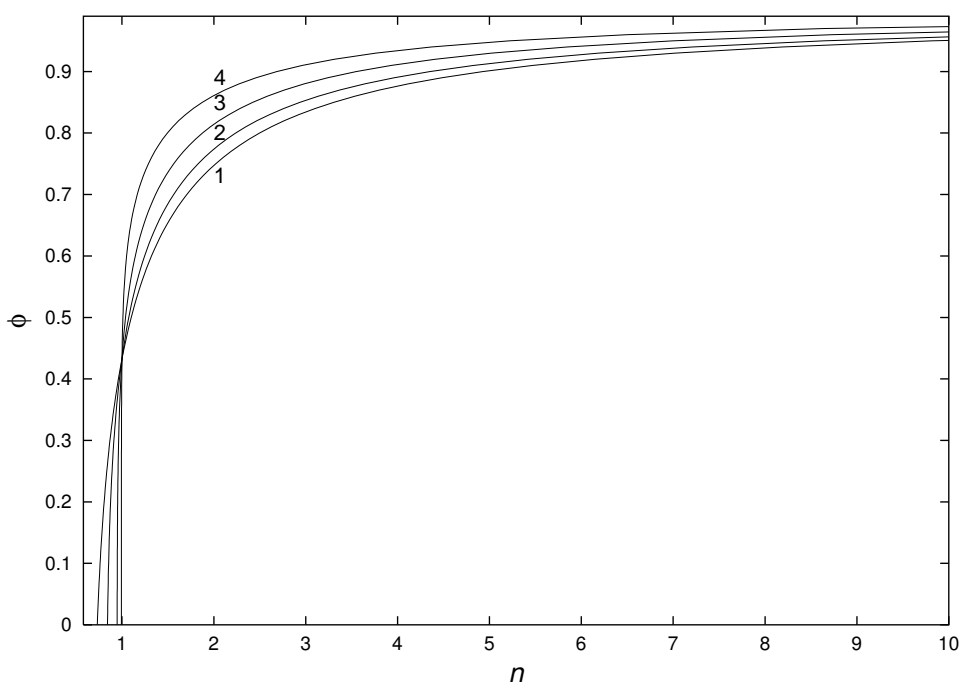

Fig. 16. Examples of $\phi(n)$ for $a=0.5, a=2, a=3 \quad a=5$ (curves 1,2, 3 and 4, respectively).

the function

$$
\tilde{w}(n)=|w(n)|_{\phi^{\prime}(n) \equiv 1}=C \exp \left[-a\left(a_{1}-\frac{a_{2} \phi(n)}{r_{m}}+\frac{a_{3} \phi^{2}(n)}{r_{m}^{2}}\right)\right]
$$

has a maximum at the point $n$ corresponding to the position of the unstable equilibrium, and rapidly decreases with decreasing $n$ (see Fig. 17). As a increases, the maximum becomes sharper, and its height increases.

It can easily be shown that

$$
\phi\left(n_{\min }\right)=0, \quad \phi(1)=\frac{r_{\min }}{r_{0}}, \quad \phi(\infty) \rightarrow 1
$$

\subsection{Influence of the Earth's rotation on alpha decay}

In the previous subsection we derived a formula for the probability distribution of the alpha decay rate, based on the classical equation for alpha particle motion within the nucleus. If we now add to this equation an external force $F(\mathbf{r}, \dot{\mathbf{r}})$, subject to inertial effects as before (see Eq. (5.14)) and, assuming that the conditions (5.2) are fulfilled, we obtain

$$
\gamma \dot{\mathbf{r}}+\frac{d V(r)}{d r}=\mathbf{F}(\mathbf{r}, \dot{\mathbf{r}})+m \xi(t)
$$



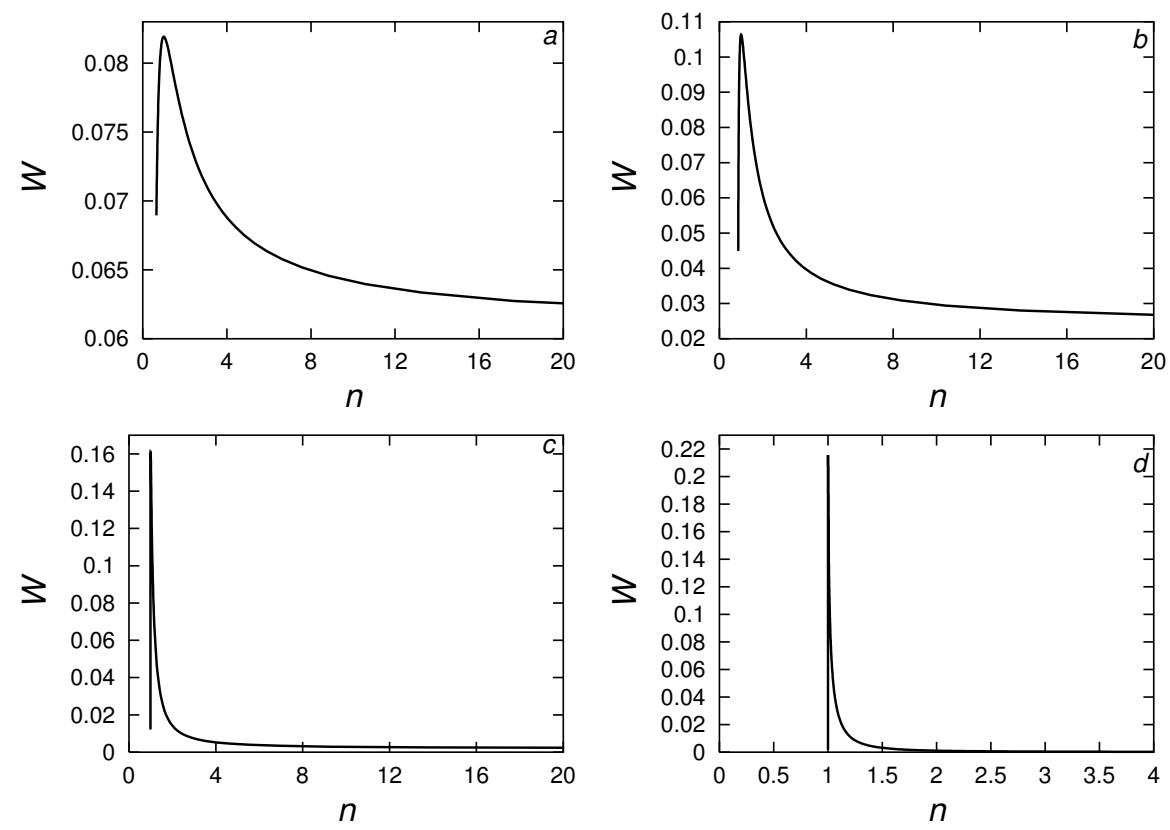

Fig. 17. Dependences of $w(n)$ for $\gamma=10^{-7}, K=10^{-5}$ and: (a) $a=0.2$; (b) $a=0.4$; (c) $a=0.8$; and (d) $a=1.6$.

where

$$
\mathbf{F}(\mathbf{r}, \dot{\mathbf{r}}) \approx \frac{\mathbf{f}_{\mathrm{cf}}}{m}=-\left\{\left(\boldsymbol{\Omega}_{1}-\frac{\mathbf{v}}{R_{1}}\right)^{2} \mathbf{r}_{1}+\left(\boldsymbol{\Omega}_{2}-\frac{\mathbf{v}}{R_{2}}\right)^{2} \mathbf{r}_{2}-2\left[\boldsymbol{\Omega}_{1} \mathbf{v}\right]\right\}+\xi(t)
$$

$\mathbf{v}$ is the velocity of the alpha particle, and the other variables are defined as before.

We now perform the same transformations as in the previous section.

$$
\gamma \dot{\mathbf{r}}+\frac{d V(r)}{d r}+\left\{\left(\boldsymbol{\Omega}_{1}-\frac{\mathbf{v}}{R_{1}}\right)^{2} \mathbf{r}_{1}+\left(\boldsymbol{\Omega}_{2}-\frac{\mathbf{v}}{R_{2}}\right)^{2} \mathbf{r}_{2}-2\left[\boldsymbol{\Omega}_{1} \mathbf{v}\right]\right\}=\xi(t)
$$

where $\left|r_{1,2}\right|$ are defined by (5.11).

The Fokker-Planck equation for the probability density $w(v)$ corresponding to Eq. (5.45) is

$$
\frac{\partial w}{\partial t}+\frac{\partial G(v)}{\partial v}=0
$$

where

$$
G(v)=-\gamma v-\frac{d V(r)}{d r}-\left[\left(\Omega_{1}-\frac{v}{R_{1}}\right)^{2} r_{1}+\left(\Omega_{2}+\frac{v}{R_{2}}\right)^{2} r_{2}-2 \Omega_{1} v\right]-\frac{K}{2} \frac{\partial w}{\partial v}
$$


The stationary solution of Eq. (5.46) under the condition of vanishing probability flow $G(v)$ is

$$
w(v)=C \exp \left\{-\frac{\gamma v^{2}}{K}+\frac{2 v}{K}\left[r_{1}\left(\Omega_{1}^{2}-\frac{\Omega_{1} v}{R_{1}}+\frac{v^{2}}{3 R_{1}^{2}}\right)+r_{2}\left(\Omega_{2}^{2}-\frac{\Omega_{2} v}{R_{2}}+\frac{v^{2}}{3 R_{2}^{2}}\right)-2 \Omega_{1} v\right]\right\}
$$

which is similar in form to Eq. (4.18). Again, the probability distribution depends on the sign of the velocity $v$. For $v>0$ it is a nonmonotonic function of $v$ (it can have several extrema), but for $v<0$ it is a monotonically decreasing function. As seen from expression (5.48), the model considered can give one, two or three extrema corresponding to the number of real roots of the cubic equation

$$
-\frac{\gamma v^{2}}{K}+\frac{2 v}{K}\left[r_{1}\left(\Omega_{1}^{2}-\frac{\Omega_{1} v}{R_{1}}+\frac{v^{2}}{3 R_{1}^{2}}\right)+r_{2}\left(\Omega_{2}^{2}-\frac{\Omega_{2} v}{R_{2}}+\frac{v^{2}}{3 R_{2}^{2}}\right)-2 \Omega_{1} v\right]=0 .
$$

These results correspond qualitatively to Shnol's experimental observations. As in the previous section, the form of the probability distribution depends on the local time, which also corresponds to Shnol's experiments.

\section{Conclusion}

We have shown that, in systems with interacting random fast and regular or random slow processes, the probability distribution for the fast processes can essentially change. The cause lies in a modulation of the fast processes by the slow ones. This phenomenon takes place for any fast random process that interacts with a slow process or processes. This is strikingly illustrated by the results presented in the book by Shnol' [35], where a great diversity of systems is described. Note, however, that Shnol' uses terminology which, in our judgment, does not adequately reflect the phenomena that he considers. To try to corroborate our view, we have considered two examples relevant to (but missing from) Shnol's book, namely, we have calculated theoretically the change in the probability distribution of the fast random component for the cases of stochastic resonance resulting from the presence of the slow harmonic forcing, and for the pendulum with a randomly vibrated suspension modelling a turbulent jet; we have also investigated two 
of Shnol's main examples, Brownian motion and $\alpha$-decay. Our calculations are consistent with Schnol's hypothesis of the universality of the phenomena described.

\section{References}

[1] I. I. Blekhman (Ed.), Selected Topics in Vibrational Mechanics, World Scientific, Singapore, 2004 .

[2] A. M. Dykhne, V. P. Kraynov, Fast and Slow Subsystems in Atomic Physics (in Russian), Azbuka, St. Petersburg, 2002.

[3] P. S. Landa, P. V. E. McClintock, Changes in the dynamical behavior of nonlinear systems induced by noise, Phys. Rep. 323 (1) (2000) 1-80.

[4] B. Van der Pol, A theory of the amplitude of free and forced triode vibrations, Radio Rev. 1 (1920) 701-710.

[5] B. Van der Pol, Selected Scientific Papers, North-Holland, Amsterdam, 1960.

[6] N. M. Krylov, N. N. Bogolyubov, Introduction to Nonlinear Mechanics, Princeton Univ. Press, Princeton, 1947.

[7] N. N. Bogolyubov, Perturbation theory in nonlinear mechanics, Sbornik Instituta Stroitel'noy Mekhaniki AN USSR (14) (1950) 9-34.

[8] N. N. Bogolyubov, Y. A. Mitropol'sky, Asymptotic Methods in the Theory of Nonlinear Oscillations, Gordon and Breach, New York, 1961.

[9] V. M. Volosov, Averaging in systems of ordinary differential equations, Russ. Math. Surv. 17 (6) (1962) 1-126.

[10] V. M. Volosov, B. I. Morgunov, Averaging Method in the Theory of Nonlinear Oscillatory Systems (in Russian), Moscow University Press, Moscow, 1971.

[11] Y. A. Mitropol'sky, The Averaging Method in Nonlinear Mechanics (in Russian), Naukova Dumka, Kiev, 1971.

[12] Y. Y. Borisov, N. M. Gynkina, Excitation of high-velocity jets by acoustic oscillations (in Russian), Akusticheskiy Zhurnal 21 (1975) 364. 
[13] Y. V. Vlasov, A. S. Ginevsky, Problem of aeroacoustic interactions, Soviet Phys. Acoustics $26(1980) 1-7$.

[14] V. Kibens, Discrete noise spectrum generated by an acoustically excited jet, AIAA J. 18 (4) (1980) 434-441.

[15] K. M. B. Q. Zaman, A. K. M. F. Hussain, Turbulence suppression in free shear flows by controlled excitation, J. Fluid Mech. 103 (1981) 133-159.

[16] M. Nallasamy, Turbulence suppression at high amplitudes of excitation: numerical and experimental study, Bull. Am. Phys. Soc. 28 (1983) 1380.

[17] M. Nallasamy, A. K. M. F. Hussain, Numerical study of the phenomenon of turbulence suppression in a plane shear layer, Turbulent Shear Flows 4 (1984) 169.

[18] Y. V. Vlasov, A. S. Ginevsky, R. K. Karavosov, Influence of the mode composition of acoustic disturbances on jet aerodynamic characteristics, Soviet Phys. Acoustics 32 (1986) 329-330.

[19] G. Raman, K. B. M. Q. Zaman, E. J. Rice, Initial turbulence effect on jet evolution with and without tonal excitation, Phys. Fluids A 1 (7) (1989) 1240-1248.

[20] H. E. Fielder, H. H. Fernholz, On management and control of turbulent shear flows, Progr. Aerospace Sci. 27 (1990) 305-387.

[21] A. S. Ginevsky, P. S. Landa, A. A. Zaikin, Self-excitation of impinging jets with regard to acoustic feedback, in: Third Intern. Congress on Air- and Structure-borne Sound and Vibration, Montreal, 1994, pp. 1191-1198.

[22] Y. V. Vlasov, A. S. Ginevsky, R. K. Karavosov, T. M. Makarenko, Turbulence suppression in subsonic jets by high-frequency acoustic excitation, Fluid Dynamics 34 (1999) 23-28.

[23] P. S. Landa, A. S. Ginevsky, Y. V. Vlasov, A. A. Zaikin, Turbulence and coherent structures in subsonic submerged jets. Control of the turbulence, Int. J. Bifurc. and Chaos 9 (2) (1999) $397-414$.

[24] P. S. Landa, Regular and Chaotic Oscillations, Springer-Verlag, Berlin, 2001.

[25] Y. V. Vlasov, A. S. Ginevsky, P. S. Landa, D. L. Samodurov, On analogy between the responce on periodical excitation of a turbulent jet and the pendulum with randomly vibrated suspension axis, J. Eng. Phys. and Thermophys. 75 (4) (2002) 90-93.

[26] P. S. Landa, Nonlinear Oscillations and Waves, URSS, Moscow, 2010. 
[27] H. Dresig, J. I. Vulfson, Dampfungtheorie bei nichtharmonischer belastung dampfung und nichtlinearitat, VDI-Bericht 1082 (1993) 141-156.

[28] P. S. Landa, P. V. E. McClintock, Vibrational resonance, J. Phys. A: Math. Gen. 33 (45) (2000) L433-L438.

[29] I. I. Blekhman, P. S. Landa, Conjugate resonances in nonlinear systems under dual-frequency forcing. vibroinduced bifurcations (in Russian), Appl. Nonlin. Dynamics 10 (1-2) (2002) 4451.

[30] J. P. Baltanás, L. López, I. I. Blekhman, P. S. Landa, A. Zaikin, J. Kurths, M. A. F. Sanjuán, Experimental evidence, numerics, and theory of vibrational resonance in bistable systems, Phys. Rev. E 67 (6) (2003) 066119.

[31] I. I. Blekhman, P. S. Landa, Conjugate resonances and bifurcations in nonlinear systems under biharmonical excitation, Int. J. of Non-linear Mechanics 39 (2004) 421-426.

[32] J. H. Jenkins, E. Fischbach, J. B. Buncher, J. T. Gruenwald, D. E. Krause, J. J. Mattes, Evidence of correlations between nuclear decay rates and Earth-Sun distance, Astropart. Phys. 32 (2009) 42-46.

[33] P. A. Sturrock, A. G. Parkhomov, E. Fischbach, J. H. Jenkins, Power spectrum analysis of LMSU (Lomonosov Moscow State University) nuclear decay-rate data: Further indication of r-mode oscillations in an inner solar tachocline, Astropart. Phys. 35 (11) (2012) 755-758.

[34] A. V. Kaminsky, S. E. Shnoll, Cosmophysical factors in the fluctuation amplitude spectrum of Brownian motion, Prog. in Phys. 3 (2010) 25-30.

[35] S. E. Shnoll, Cosmophysical Factors in Stochastic Processes, American Research Press, Rehoboth NM, 2012.

[36] P. S. Landa, Mechanism of stochastic resonance, Doklady Physics 49 (12) (2004) 706-709.

[37] P. Landa, V. Ushakov, J. Kurths, Rigorous theory of stochastic resonance in overdamped bistable oscillators for weak signals, Chaos, Solitons \& Fractals 30 (3) (2006) 574-578.

[38] B. B. Mandelbrot, Fractals - Form, Chance and Dimension, Freeman, San Francisco, 1977.

[39] B. B. Mandelbrot, The Fractal Geometry of Nature, Freeman, Oxford, 1982. 
[40] P. S. Landa, V. A. Vlasov, Analytical consideration of the effect of cosmic factors on the velocity fluctuations of Brownian particles (in Russian), Prikladnaya Nelineynaya Dinamika 19 (2011) 56-88.

[41] S. E. Shnol', P. S. Landa, V. A. Vlasov, The effect of cosmic factors on the alpha decay velocity, Vestnik nauchno-tekhnicheskogo razvitiya 2 (2011) 1.

[42] R. Benzi, A. Sutera, A. Vulpiani, The mechanism of stochastic resonance, J. Phys. A: Math. Gen. 14 (11) (1981) L453-L457.

[43] G. Nicolis, C. Nicolis, Stochastic aspects of climate transitions and additive fluctuations, Tellus 33 (3) (1981) 225-234.

[44] R. L. Stratonovich, Topics in the Theory of Random Noise, vol. 1, Gordon and Breach, New York, 1963.

[45] R. L. Stratonovich, Topics in the Theory of Random Noise, vol. 2, Gordon and Breach, New York, 1967.

[46] P. S. Landa, Y. I. Neimark, P. V. E. McClintock, Changes in the effective parameters of averaged motion in nonlinear systems subject to noise, J. Stat. Phys. 125 (3) (2006) 593-620.

[47] Y. V. Vlasov, A. S. Ginevsky, Acoustic modification of the aerodynamic characteristics of a turbulent jet, Fluid Dynamics 2 (4) (1967) 93-96.

[48] Y. V. Vlasov, A. S. Ginevsky, Generation and suppression of turbulence in an axisymmetric turbulent jet in the presence of an acoustic influence (in Russian), Mekhnika Zhidkosti i Gaza 6 (1973) 37-43.

[49] G. Raman, E. J. Rice, R. R. Mankbadi, Saturation and a limit of jet mixing enhancement by single frequency plane wave excitation: experiment and theory (from 1st Nat. Fluid Dyn. Congr.), in: Collected Papers, AIAA/ASSME/SIAM/APS, Cincinnati, 1988, pp. 1000-1007.

[50] Y. V. Vlasov, A. S. Ginevsky, R. K. Karavosov, T. M. Makarenko, On change of the sign of high-frequency acoustic action on a turbulent jet with the increase of excitation, J. Eng. Phys. and Thermophys. 1 (2001) 8-9.

[51] A. Ginevsky, Control of coherent structures and aero-acoustic characteristics of subsonic and supersonic turbulent jets, in: IUTAM Symposium "The Active Control of Vibration", Proceedings, Bath, IUTAM, 1994, pp. 203-207. 
[52] P. S. Landa, V. G. Ushakov, Manifestation of the stochastic resonance in submerged jets under an acoustic action, JETP Lett. 86 (5) (2007) 304-307.

[53] M. Nallasamy, A. K. M. F. Hussain, Effects of excitation on turbulence levels in a shear layer, J. Fluids Eng. Trans. ASME 111 (1) (1989) 102-104.

[54] P. S. Landa, A. A. Zaikin, Noise-induced phase transitions in a pendulum with a randomly vibrating suspension axis, Phys. Rev. E 54 (4) (1996) 3535-3544.

[55] P. S. Landa, Universality of oscillation theory laws. Types and role of mathematical models, Discrete Dynamics in Nature and Society 1 (2) (1997) 99-110.

[56] P. S. Landa, A. A. Zaikin, M. G. Rosenblum, J. Kurths, On-off intemitteney phenomena in a pendulum with a randomly vibrating suspension axis, Chaos, Solitons \& Fractals 9 (1-2) (1998) 157-169.

[57] N. Platt, E. A. Spiegel, C. Tresser, On-off intermittency: a mechanism for bursting, Phys. Rev. Lett. 70 (3) (1993) 279-282.

[58] H. Fujisaka, T. Yamada, A new intermittency in coupled dynamical systems, Progr. Theor. Phys. 74 (4) (1985) 918-921.

[59] J. F. Heagy, N. Platt, S. M. Hammel, Characterization of on-off intermittency, Phys. Rev. E 49 (2) (1994) 1140-115.

[60] H. G. Schuster, Deterministic Chaos, Physik-Verlag, Weinheim, 1984.

[61] Y. L. Klimontovich, Statistical Physics, Harwood, New York, 1986.

[62] A. M. Prokhorov (ed.), Physical Encyclopædia (in Russisn), 1988, pp. 63-64.

[63] W. S. C. Williams, Nuclear and Particle Physics, Clarendon, Oxford, 1991.

[64] L. S. Pontryagin, A. A. Andronov, A. A. Witt, On the statistical treatment of dynamical systems (in Russian; translated by J. Barbour in Noise in Nonlinear Dynamical Systems, eds. F. Moss and P. V. E. McClintock, vol. 1, Cambridge University Press, 1989, pp 329-348), Zh. Eksp. Teor. Fiz. 3 (1933) 165-180.

[65] P. S. Landa, R. L. Stratonovich, On the theory of fluctuational transitions from one stationary state to another for different systems (in Russian), Vestnik MGU III (1) (1962) 33-45. 
[66] E. B. Dynkin, Theory of Markov Processes (reprint of 1961 edition), Dover, Mineola NY, 2006. 